Check for updates

Cite this: Mater. Chem. Front., 2019, 3, 1973

Received 1st July 2019,

Accepted 2nd August 2019

DOI: $10.1039 / c 9 q m 00428 a$

rsc.li/frontiers-materials

\section{Stimuli-responsive nanocarriers constructed from pillar[n]arene-based supra-amphiphiles}

\author{
Tangxin Xiao, (D) *a Lijie Qi, ${ }^{a}$ Weiwei Zhong, ${ }^{a}$ Chen Lin, ${ }^{* b}$ Ruibing Wang (D) ${ }^{c}$ and \\ Leyong Wang (iD) ab
}

\begin{abstract}
Supra-amphiphiles assembled by macrocycle-based host-guest interactions have received considerable attention in recent years due to their dynamic properties and wide applications. Pillar[ $n]$ arenes are a unique family of macrocycles which exhibit outstanding host-guest behavior. Developing water-soluble pillar[n]arene (WP[n]) based supra-amphiphiles is an effective way to introduce this special macrocycle into functional nanomaterials through controlled self-assembly. Specifically, WP[n]-based supraamphiphiles can further form different nanomaterials, such as micelles, vesicles, and other types of nanoparticles (NPs), which may become good candidates for nanocarriers of payloads. Herein, we highlight recent significant achievements in functional nanocarriers fabricated from supra-amphiphiles based on WP[n]s. By showing a series of examples, the preparation strategies, characterization, and stimuli-responsive behaviors of these nano-sized assemblies based on WP[n] derivatives are described. Furthermore, some important applications of WP[n]-based nanocarriers, such as drug/gene/protein delivery, photodynamic/photothermal therapy, and optical materials, are discussed in detail. Finally, future challenges and research directions in this rapidly evolving field are outlined.
\end{abstract}

${ }^{a}$ School of Petrochemical Engineering, Advanced Catalysis and Green Manufacturing Collaborative Innovation Center, Changzhou University, Changzhou, 213164, China. E-mail: xiaotangxin@cczu.edu.cn

${ }^{b}$ Key Laboratory of Mesoscopic Chemistry of MOE, School of Chemistry and Chemical Engineering, Nanjing University, Nanjing, 210023, China. E-mail: linchen@nju.edu.cn

${ }^{c}$ State Key Laboratory of Quality Research in Chinese Medicine, Institute of Chinese Medical Sciences, University of Macau, Taipa, Macau SAR 999078, China. E-mail: rwang@um.edu.mo

\section{Introduction}

The combination of supramolecular chemistry and nanotechnology has greatly promoted the development of nano-sized assemblies, which are usually employed as promising nanomaterials for controlled release, drug delivery, bio-imaging, and light harvesting. ${ }^{1-3}$ Inspired by liposomes spontaneously formed from amphiphilic phospholipids in aqueous media in Nature, scientists are seeking to construct more functional

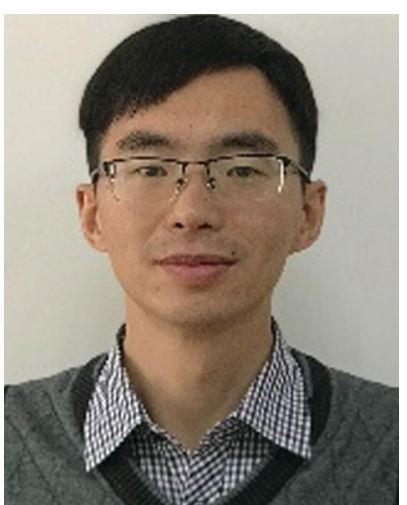

Tangxin Xiao
Tangxin Xiao is a Lecturer at the School of Petrochemical Engineering, Changzhou University. He studied chemistry at Nanjing University where he received his PhD degree under the supervision of Prof. Leyong Wang in 2014. He had been to the University of Cambridge as a visiting PhD student supervised by Prof. Oren Scherman in 2013. After postdoctoral research on fine chemicals at the Zhejiang University-NHU Company United R\&D Center, he

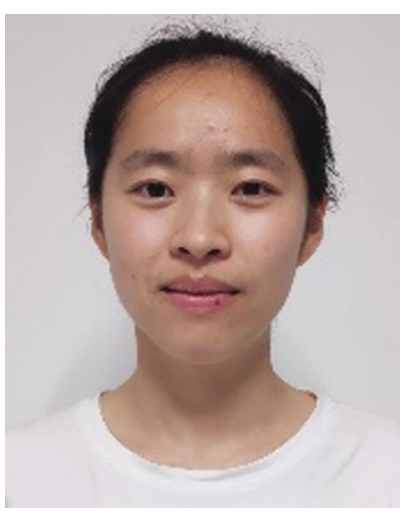

Lijie Qi
Lijie Qi was born in Henan province, China. She obtained her B.Sc. degree from Shangqiu Normal University in 2018. She is currently pursuing her Master's degree under the supervision of Dr Tangxin Xiao and Prof. Leyong Wang at Changzhou University. Her research interests are focused on quadruple hydrogen bonding supramolecular polymers.

joined Changzhou University in 2017. His current research interests concern supramolecular self-assembly and smart materials. 
nanoassemblies from various synthetic amphiphiles. ${ }^{4}$ Conventionally, amphiphiles refer to molecules containing both hydrophobic and hydrophilic units that are linked by covalent chemical bonds. ${ }^{5}$ Amphiphiles generally self-assemble into well-ordered architectures with different shapes, such as nanosheets, nanorods, and nanospheres (micelles, vesicles, or other forms of NPs). Investigation of these nanoassemblies not only discloses the law of self-assembly but also provides more and more applications in many areas including detergents, templated synthesis of inorganic nanostructures, and drug/ gene delivery etc.

In contrast to covalent-bond based amphiphiles, supraamphiphiles pioneered by Zhang and co-workers take advantage of supramolecular chemistry, in which the hydrophobic and hydrophilic parts are usually connected by non-covalent interactions, greatly simplifying the otherwise complexed synthetic process of amphiphilic species. ${ }^{6-9}$ Among various supramolecular interactions, macrocyclic host-guest recognitions are widely used to construct supra-amphiphiles. ${ }^{10}$ For example, Huang and co-workers summarized the recent advances in macrocyclic host-based supra-amphiphiles. ${ }^{11}$ The reversibility of macrocyclic host-guest recognitions often endows the obtained nanocarriers with controllable assembly/disassembly ability and excellent stimuli-responsive properties, which may realize their potentials in controlled cargo release and other smart functionalities. Furthermore, various supramolecular macrocyclic hosts have been continuously developed, greatly improving the vitality of this research hotspot. ${ }^{12}$ For example, water-soluble macrocycles, ${ }^{13}$ such as cyclodextrins, ${ }^{14-16}$ cucurbit $[n]$ urils, $^{17-21}$ sulfonatocalixarenes, ${ }^{22-25}$ and hydrophilic group modified crown ethers, ${ }^{26,27}$ are employed to fabricate a variety of supra-amphiphiles.

Pillar $[n]$ arenes are a relatively new family of macrocyclic hosts developed in 2008 , following the above mentioned supramolecular macrocycles. ${ }^{28-36}$ The interesting pillar-shape architectures of pillar[ $n]$ arenes endow them with the ability to bind with a variety of guest molecules. ${ }^{37}$ As a result, pillar[ $\left.n\right]$ arenes exhibit significant potentials in building various functional materials, ${ }^{38}$ such as dynamic organogels or hydrogels, ${ }^{39-45}$ functional supramolecular polymers, ${ }^{46-52}$ transmembrane

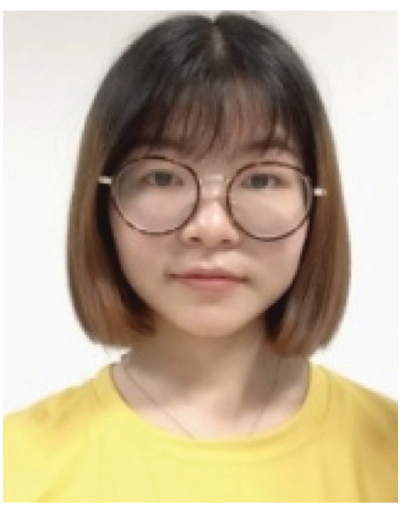

Weiwei Zhong
Weiwei Zhong was born in Jiangsu, China. She received her B.Sc. degree in Changzhou University in 2017. She is currently pursuing her M.Sc. degree under the supervision of Prof. Leyong Wang and $\mathrm{Dr}$ Tangxin Xiao at Changzhou University. Her research interests are focused on quadruple hydrogen bonding supramolecular polymers.

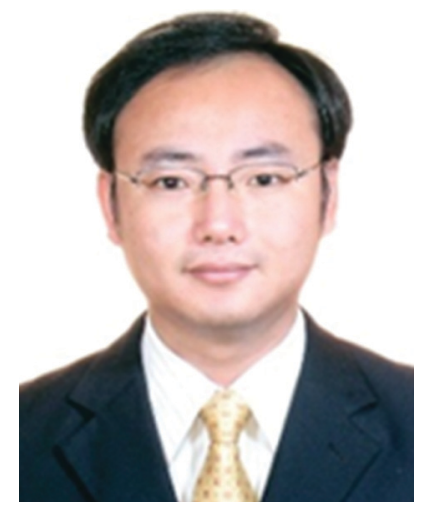

Chen Lin
Chen Lin was born in Jiangsu in China in 1979. He obtained his B.Sc. from Nankai University in China in 2001 and $P h D$ from SUNY at Stony Brook in USA in 2005. Afterwards, he joined Nanjing University as an Assistant Professor in 2006. Currently he is an Associate professor at Nanjing University. His research interests are in the areas of supramolecular chemistry, molecular recognition, and smart materials.

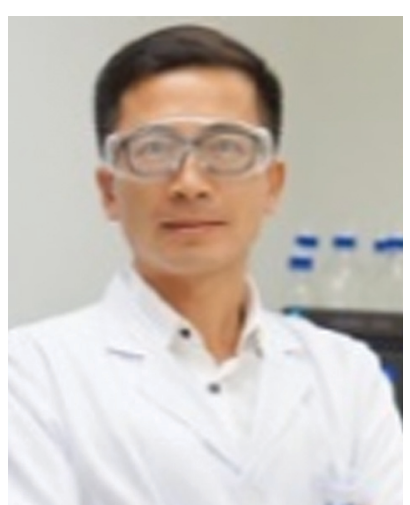

Ruibing Wang
Ruibing Wang received his B.Sc. degree in Chemistry from Jilin University in 2002 and $P h D$ degree in Organic Chemistry from Queen's University (Canada) in 2007. After a brief stay as a Postdoctoral Fellow at Queen's University, he moved to the National Research Council of Canada as a Research Associate for about two years. Between 2009 and 2014, he worked as a Senior Scientist at BTG plc, Canada. He joined the University of Macau as an Assistant Professor in Pharmaceutical Sciences in late 2014, and was recently promoted to Associate Professor. His current research interests are focused on supramolecular medicine and pharmaceutics.

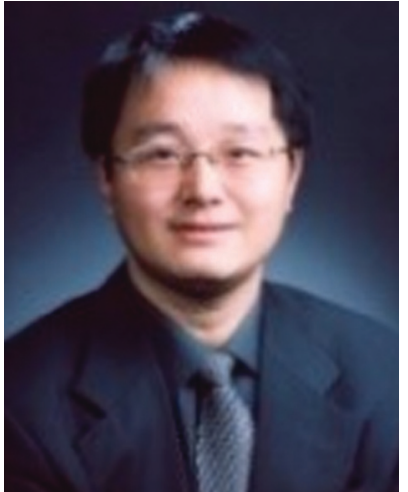

Leyong Wang
Leyong Wang holds a PhD in Chemistry from Nanjing University in 2000. After research stays at the Institute of Chemistry (CAS), the University of Burgundy, and Mainz University from 2000 to 2004, he joined the ErlangenNürnberg University as an AvH Fellow working with Professor John A. Gladysz. Since 2006, he has been specially appointed as the professor of organic and material chemistry at NJU. He is also a joint professor of

Changzhou University since 2017. His current research interests are focused on supramolecular systems ranging from topological molecules to dynamic materials. 


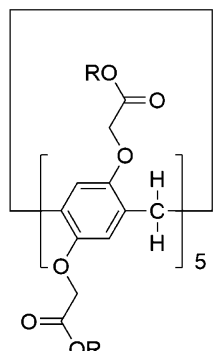

WP5a : $\mathrm{R}=\mathrm{Na}^{+}$

WP5b : $\mathrm{R}=\mathrm{NH}_{4}^{+}$
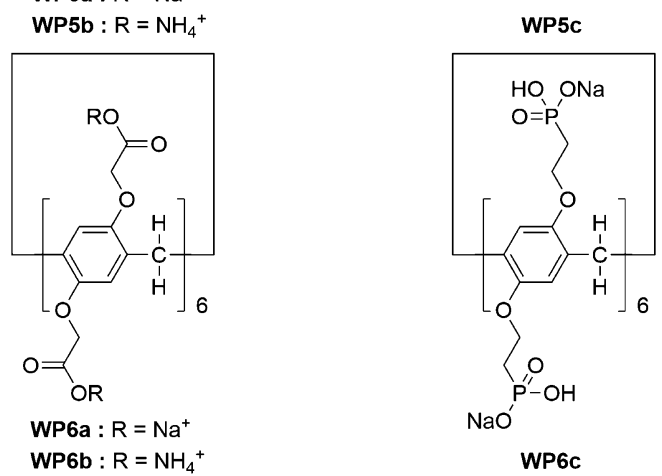

Scheme 1 Chemical structures of some selected pillar[n]arenes discussed in this review.

channels, ${ }^{53}$ nonporous adaptive crystals, ${ }^{54-57}$ and other nanomaterials. ${ }^{58,59}$ Meanwhile, the development of water-soluble pillar $[n]$ arene (WP $[n])$ based supra-amphiphiles has also progressed rapidly in recent years due to the simplicity of modification of the pillar[n]arene framework. ${ }^{60-63}$ Notably, the size of $\mathrm{WP}[n] \mathrm{s}$ varies with $n$ value (most commonly $n=5$ or 6 ), resulting in great possibilities to construct various supra-amphiphiles with different sized polar heads. For example, the chemical structures of some $\mathrm{WP}[n] \mathrm{s}$ discussed in this review are displayed in Scheme 1 . Two main types of $\mathrm{WP}[n]$-based supraamphiphiles are well-known: tadpole-like and bola-like supraamphiphiles (Fig. 1). In addition, there are gemini-type and polymer-based supra-amphiphiles, which are also discussed in the following part.

A nanocarrier generally refers to a nanomaterial being used as a carrier for another cargo, such as a dye or a drug. Although some WP $[n]$-based supra-amphiphiles have been discussed in some recent reviews, ${ }^{11,60}$ we herein mainly focus on the

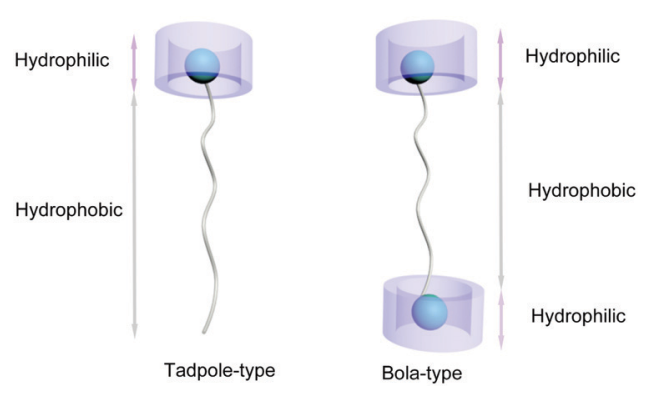

Fig. 1 Cartoon illustration of two representative supra-amphiphiles based on WP[n]: tadpole-type (left) and bola-type (right). "payload loading and responsive release" of these nanoassemblies. Such nanoassemblies include various types of nanostructures, such as micelles, vesicles, and other forms of NPs, all of which could serve as carriers for cargoes. Notably, a very recent review concerning supramolecular nanotheranostics based on pillar $[n]$ arenes was reported. ${ }^{64}$ However, this mainly focuses on hybrid porous materials and some supramolecular amphiphilic systems for only drug delivery. In this review, we will first describe the construction methods and stimuli-responsive properties of these smart nanocarriers self-assembled from WP $[n]$-based supra-amphiphiles. Subsequently, we will describe several promising applications of these nanocarriers. According to different applications, WP $[n]$-based nanocarriers can be categorized into the following types: (a) nanocarriers for drug/ gene/protein delivery; (b) nanocarriers for photodynamic/ photothermal therapy and (c) nanocarriers as optical materials. Finally, the developing challenges and future perspectives of $\mathrm{WP}[n]$-based nanocarriers are discussed as well.

\section{WP[n]-based supra-amphiphiles for controlled assembly and disassembly upon exposure to external stimuli}

Self-assembly of supra-amphiphiles can generate nano-sized assemblies in an aqueous solution. The assembly/disassembly of such nanoassemblies could well be controlled by host-guest interactions. By introducing responsive chemical groups to the host or guest molecules, the reversibility of such host-guest interactions could be realized upon exposure to environmental stimuli. In terms of $\mathrm{WP}[n], \mathrm{pH}$ responsiveness is very easily adopted via modification of the backbone of $\mathrm{WP}[n]$. However, it is relatively complicated to introduce other responsive units into the WP $[n]$ host backbones. In contrast, the modification of guest molecules is more diverse and straightforward, providing the possibilities to realize various stimuli-responsiveness, such as photo-, redox-, glutathione (GSH)-responsiveness, etc. The responsiveness generates a remarkable impact on whether a nanoassembly can be constructed with a certain functionality. In this section, we will give some examples of $\mathrm{WP}[n]$-based nanocarriers that respond to different external stimuli.

\section{1 pH-responsive nanocarriers fabricated by WP $[n]$-based supra-amphiphiles}

Considering the broad range of $\mathrm{pH}$ gradients in the biological system, the construction of $\mathrm{pH}$ responsive supra-amphiphiles for further self-assembly is very interesting. In 2012, Huang and co-workers prepared vesicles from a WP[6] (WP6a, Fig. 2) and a pyridinium salt-based guest (G1). ${ }^{65}$ The water solubility of WP6a was achieved by connecting carboxylate units to both sides of the host backbone. WP6a strongly bound G1 in water to form a supra-amphiphile through host-guest interaction. Transmission electron microscopy (TEM) showed that nanotubes were formed from G1. However, when 1 equiv. of WP6a was added, supramolecular vesicles were obtained. Interestingly, these nanotubes appeared again after the $\mathrm{pH}$ of the mixture 


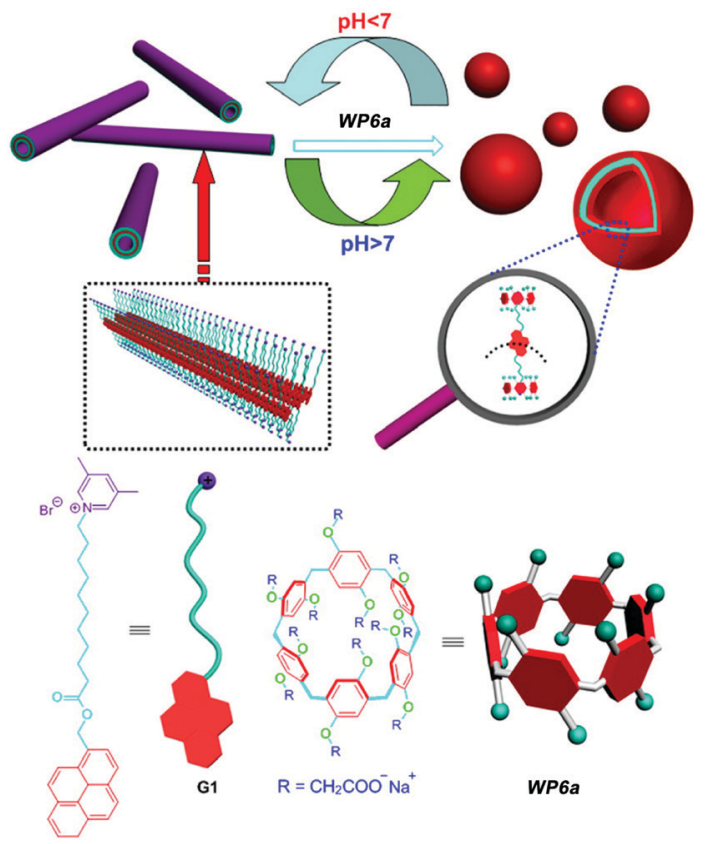

Fig. 2 Chemical structures of WP6a and G1 and their cartoon representation of reversible transformations between vesicles and nanotubes. Reproduced with permission from ref. 65. Copyright 2012, American Chemical Society.

was changed to 6.0. The protonation of the carboxylate groups on WP6a reduced the solubility of WP[6], resulting in the disruption of the supra-amphiphile. As a result, the assembly/ disassembly of the host-guest complex could be reversibly controlled by $\mathrm{pH}$, leading to reversible morphological changes between supramolecular vesicles and nanotubes.

In another study, Huang and co-workers constructed a pH-responsive vesicle from WP6a and a paraquat-based molecule. ${ }^{66}$ In this example, the paraquat guest was also amphiphlic, which contained a hydrophilic 4,4'-bipyridinium unit and a hydrophobic long alkyl chain. The paraquat guest itself formed micelles in water. Upon addition of WP6a, the generation of supramolecular vesicles with low curvature was achieved. This might be due to the electrostatic repulsion and the steric hindrance generated by the insertion of WP6a macrocycles. Similar to the previous example, the changes between micelles and supramolecular vesicles were realized by changing the $\mathrm{pH}$ of the solution. Therefore, the controlled release of calcein was successfully achieved owing to the $\mathrm{pH}$ induced transformation. Moreover, they further reported the first WP[7] molecule and studied its binding behavior with paraquat derivatives, which was controlled by $\mathrm{pH}^{67}$

\subsection{Photo-responsive nanocarriers fabricated by WP $[n]$-based supra-amphiphiles}

Light is a very attractive external stimulus because of its remote, clean, rapid, and noninvasive nature. Azobenzene is a representative photo-responsive motif, which has been employed in many supramolecular systems. In 2014, Huang and co-workers prepared a supra-amphiphile formed by a WP[6] (WP6b, Fig. 3) and an azobenzene-based molecule (G3). ${ }^{68}$ The amphiphilic

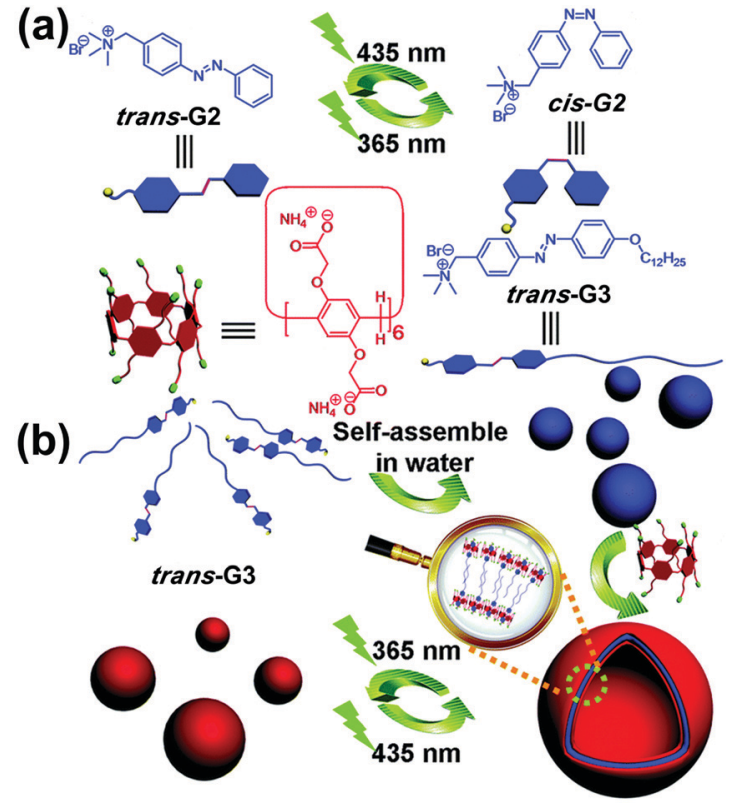

Fig. 3 (a) Chemical structures and cartoon pictures of G2, G3, and WP6b. (b) Cartoon representation of the photo-sensitive transformation based on WP6b and G3. Reproduced with permission from ref. 68. Copyright 2014, the Royal Society of Chemistry.

guest molecule trans-G3 alone formed NPs, as evidenced by TEM. Upon addition of WP6b, supramolecular vesicles were obtained. The vesicles were sensitive to UV/vis light irradiations due to the incorporation of photo-responsive azobenzene groups. The disassembly mechanism is shown as follows: upon UV light irradiation, trans-G3 turned into cis-G3, which dethreaded from WP6b. Thus, the arrangement of the supraamphiphiles was changed, leading to the reappearance of solid NPs. Therefore, reversible transformations between supramolecular vesicles and NPs were realized owing to the photoresponsive property of the azobenzene guest. In a subsequent study, Huang and co-workers further prepared a UV-responsive supra-amphiphile by employing WP6a and a UV-responsive guest containing a 2-nitrobenzyl ester moiety. ${ }^{69}$

Similar to azobenzene, spiropyran is also an interesting photo-responsive motif. The reversible transformation of spiropyran from a ring-closed form to ring-open merocyanine can be well controlled by UV/vis light irradiation alternatively. In 2018, Yin and co-workers successfully constructed a photocontrolled system by employing a spiropyran derivative (G4) and WP5b (Fig. 4). ${ }^{70}$ Guest $\mathbf{G 4}$ could form a supra-amphiphile with WP5b. Generally, $\mathbf{G 4}_{\mathbf{M}}$ and $\mathbf{G} \mathbf{4}_{\mathbf{s}}$ coexisted in water as solid nanospheres. Upon irradiating at $520 \mathrm{~nm}$, the nanospheres did not change although the hydrophilic $\mathbf{G 4}_{\mathbf{M}}$ was transformed to amphiphilic $\mathbf{G 4}_{\mathbf{s}}$. However, when $\mathbf{W P 5} \mathbf{b}$ was added into this coexistent system, supra-amphiphilic WP5b $\supset \mathbf{G 4}_{\mathbf{s}}$ and hydrophilic WP5b $\supset \mathbf{G 4}_{\mathbf{M}}$ were formed simultaneously. As a result, nanospheres and supramolecular vesicles were obtained, respectively. When the solution was exposed at $520 \mathrm{~nm}$, the NPs could be changed into vesicles due to the total change of the hydrophilic WP5b $\supset \mathbf{G 4}_{\mathbf{M}}$ to supra-amphiphilic WP5b $\supset \mathbf{G 4}_{\mathbf{s}}$. 

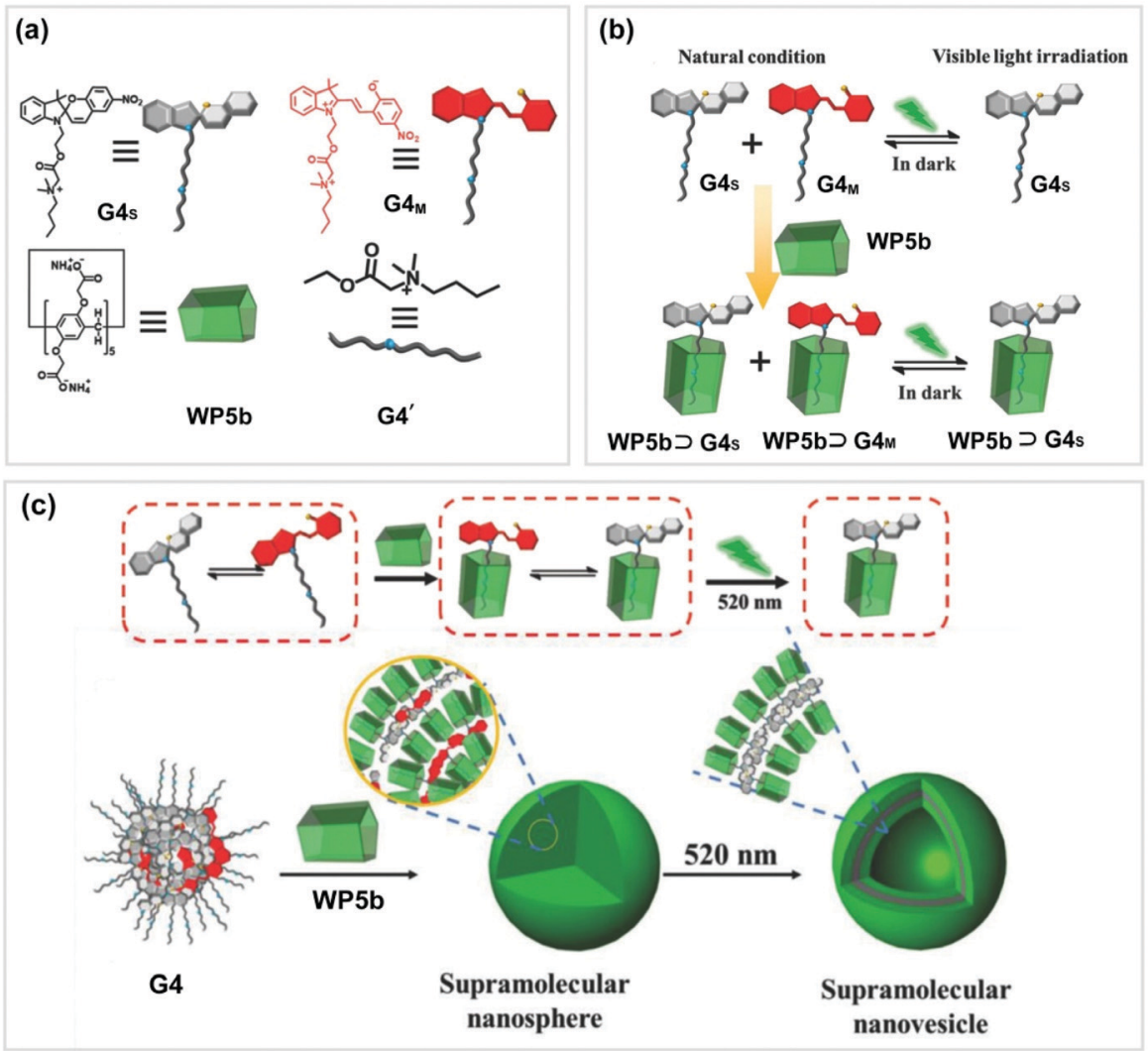

Fig. 4 (a) Chemical structures and cartoon pictures of WP5b and G4. (b) Cartoon illustration of the photoresponsive system (WP5b $\supset$ G4). (c) Transformation of the assemblies of WP5b $\supset$ G4. Reproduced with permission from ref. 70. Copyright 2018, Wiley Publishers.

This novel photoresponsive system might have potential applications in smart supramolecular materials.

The anthracene group is another widely used photoactive motif. In 2016, $\mathrm{Hu}$, Wang and co-workers fabricated a photodegradable bola-type supra-amphiphile from an anthracene-containing guest (Fig. 5). ${ }^{71}$ The guest (G5) contains 9,10-dialkoxyanthracene units and trimethylammonium terminals. G5 can form large, welldefined assemblies upon addition of WP5a. The best molar ratio of G5 and WP5a for the assembly was 6:1, which was measured by optical transmittance. The TEM pictures displayed a hollow spherical structure. The anthracene group could capture singlet oxygen and produce stable endoperoxides under UV light, resulting in the formation of anthraquinone compounds and relevant alkanols. Upon irradiation under UV light, G5 could slowly degrade, but its decomposition rate was significantly enhanced when complexed with WP5a. As a result, the obtained vesicles were disassembled. Furthermore, this nanocarrier also showed dual stimuli-responsiveness to $\mathrm{pH}$ and visible light irradiation, by loading a photosensitizer Eosin Y (ESY), suggesting a potential application for photo-sensitive therapy.

\subsection{Redox-responsive nanocarriers fabricated by WP $[n]$-based supra-amphiphiles}

Among various external stimuli, a redox-responsive process is of particular interest. For example, by changing the membrane potential, redox-responsive assemblies can be accessed via initiating

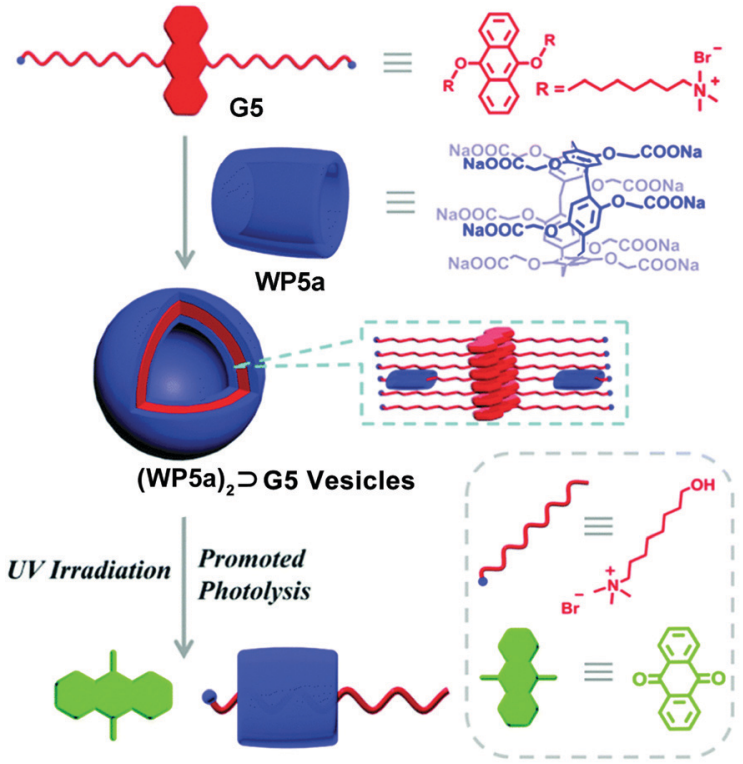

Fig. 5 Cartoon illustration of the photo-active nanocarriers based on WP5a and an anthracene derivative guest. Reproduced with permission from ref. 71. Copyright 2016, the Royal Society of Chemistry.

lipid bilayer activities in a membrane system. In 2015, Huang and co-workers reported a redox-responsive system assembled from a supra-amphiphile constituted by a neutral WP[5] (WP5d) and a 


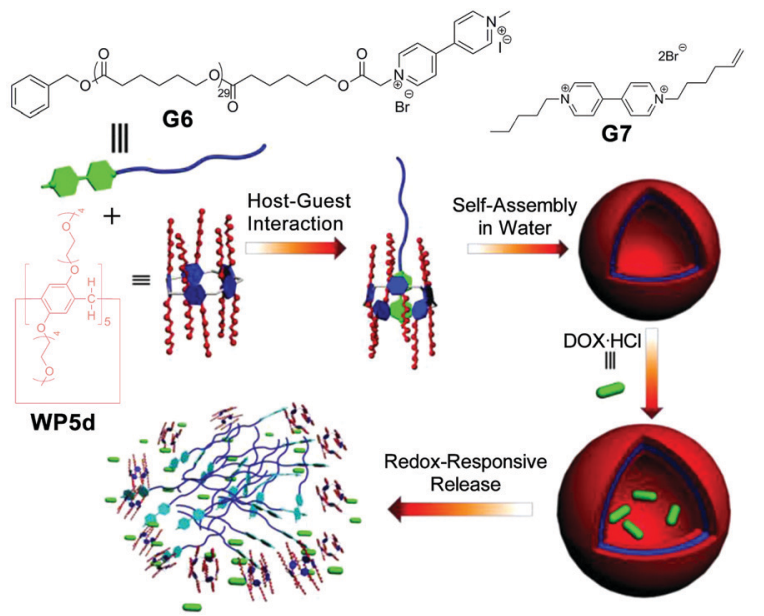

Fig. 6 Cartoon representation of the preparation of polymeric vesicles and the process of redox-controlled release. Reproduced with permission from ref. 72. Copyright 2015, American Chemical Society.

paraquat-based polymer (Fig. 6). ${ }^{72}$ They first studied the complexation between WP5d and G7 via ${ }^{1} \mathrm{H}$ NMR spectroscopy, which demonstrated that the supra-amphiphile was indeed formed in aqueous media. Moreover, a binding constant of $(4.2 \pm 0.3) \times$ $10^{4} \mathrm{M}^{-1}$ between WP5d and $\mathbf{G} 7$ was determined via isothermal titration calorimetry (ITC). They further used cyclic voltammetry to explore the redox-responsiveness of the host-guest system. After that, WP5d and polymer G6 were further employed to fabricate redox-responsive polymeric vesicles. TEM showed that the aggregates displayed a spherical morphology. Moreover, dynamic light scattering (DLS) also revealed that the size of the aggregates was similar to that observed under TEM. Upon addition of $\mathrm{Na}_{2} \mathrm{~S}_{2} \mathrm{O}_{4}$ as a reducing agent, the vesicles underwent a dramatic morphological change. Furthermore, they used anticancer drug doxorubicin hydrochloride (DOX.HCl) as a model drug to test the controlled release functions of the vesicle. The DOX-loaded vesicles displayed a minimal payload release (less than $30 \%$ ) without a stimulus, but a much faster release process in the presence of $\mathrm{Na}_{2} \mathrm{~S}_{2} \mathrm{O}_{4}$, which indicated that this nanocarrier could serve as a redox-responsive drug delivery system (DDS).

Very recently, Yang and co-workers fabricated an interesting amphiphilic pillar[5]arene-based pseudo[1] rotaxane with a dynamic redox-responsive disulfide bond in the self-included linker. ${ }^{73}$ These pseudo[1]rotaxane molecules could self-assemble into supramolecular vesicles in aqueous media, which was disrupted in the presence of GSH. This nanocarrier system is capable of encapsulating DOX with a high loading efficiency to realize controlled drug delivery. This is the first example of employing a pseudo[1] rotaxane structure to protect a vulnerable responsive bond in drug delivery systems, which provides a new possibility for the application of functional nanocarriers in precise cancer therapy.

\subsection{Multi-stimuli responsive nanocarriers fabricated by $\mathrm{WP}[n]$-based supra-amphiphiles}

In recent years, nanocarriers with multi-stimuli responsiveness have been undergoing extensive investigations due to their flexibility and intelligence geared for various applications. In 2015, Huang and co-workers prepared a supramolecular system based on a WP[7] (WP7) and an azobenzene derivative (Fig. 7) ${ }^{74}$ This novel system showed both thermo- and photoresponsiveness in aqueous media. WP7 is thermo-responsive due to its functionalization with 14 triethylene oxide groups, which endow the system with a lower critical solution temperature (LCST) behavior. Therefore, WP7 can be dissolved in water below the LCST but can become turbid above the LCST. Moreover, the polymeric azobenzene guest G8 provides photoresponsiveness. Notably, G8 could form NPs in water by itself. Subsequently, they employed WP7 and G8 to successfully fabricate a WP[7]-based polypseudorotaxane, which could further form vesicles. Therefore, the changes between supramolecular polymeric vesicles and NPs could easily be controlled by UV/vis light or temperature. Finally, the polymeric vesicles were used for the dual-stimuli responsive release of calcein molecules. This example displayed a new strategy to combine polymer chemistry with $\mathrm{WP}[n]$ to create smart nanomaterials. In a follow-up study, they fabricated a dual-thermoresponsive supramolecular vesicle from a supra-amphiphile based on the $\mathrm{WP}[10] /$ paraquat, which could respond to both heating and cooling. ${ }^{75}$ The dual-thermoresponsive properties come from the multiple triethylene oxide groups on the host and the poly( $N$-isopropylacrylamide) backbone in the polymeric guest. These nanocarriers were also employed for calcein release controlled by temperature variation.

Following the above examples, various dual-stimuli responsive nanocarriers based on $\mathrm{WP}[n]$ have been continuously constructed. For example, photo and gas dual-responsive supramolecular vesicles were constructed from a WP[6] and an azobenzene derivative by the Yu group. ${ }^{76}$ Oxidation and thermo dual-responsive polymeric vesicles were also successfully fabricated from ferrocene-WP[6]-based polymeric amphiphiles by the Wang group. ${ }^{77}$

More interestingly, Du and co-workers constructed a supramolecular nanocarrier from WP[6]-based supra-amphiphiles showing responsiveness to five stimuli, including GSH, $\mathrm{pH}$, $\mathrm{CO}_{2}, \mathrm{Zn}^{2+}$, and hexanediamine (HDA). ${ }^{78}$ The guest molecule was a disulfide-linked benzimidazolium amphiphile, which could not form vesicles by itself in aqueous media. TEM images displayed that the freeze-dried guest-WP6a aggregates showed a vesicle structure of about $165 \mathrm{~nm}$. The dye molecule $\mathrm{Ru}(\text { bipy })_{3} \mathrm{Cl}_{2}$ was employed as a model payload. Upon addition of GSH, most of the dye was released owing to the breakage of the disulfide bond, which disrupted the integrity of the nanocarriers. Upon decreasing the $\mathrm{pH}$ value to 5.0, the cargo was also released from the nanocarriers with a release efficiency of about $70 \%$. When treated with $\mathrm{CO}_{2}, 45 \%$ release efficiency was also achieved. The $\mathrm{Zn}^{2+}$ ion could also serve as a trigger for cargo release due to its chelation behavior with the carboxylate groups of WP6a. Finally, as a competitive guest, HDA was also used as a triggering stimulus. This is a rare example of $\mathrm{WP}[n]$-based nanocarriers with responsiveness to five external stimuli, which may meet different requirements for controlled payload release in different applications. 


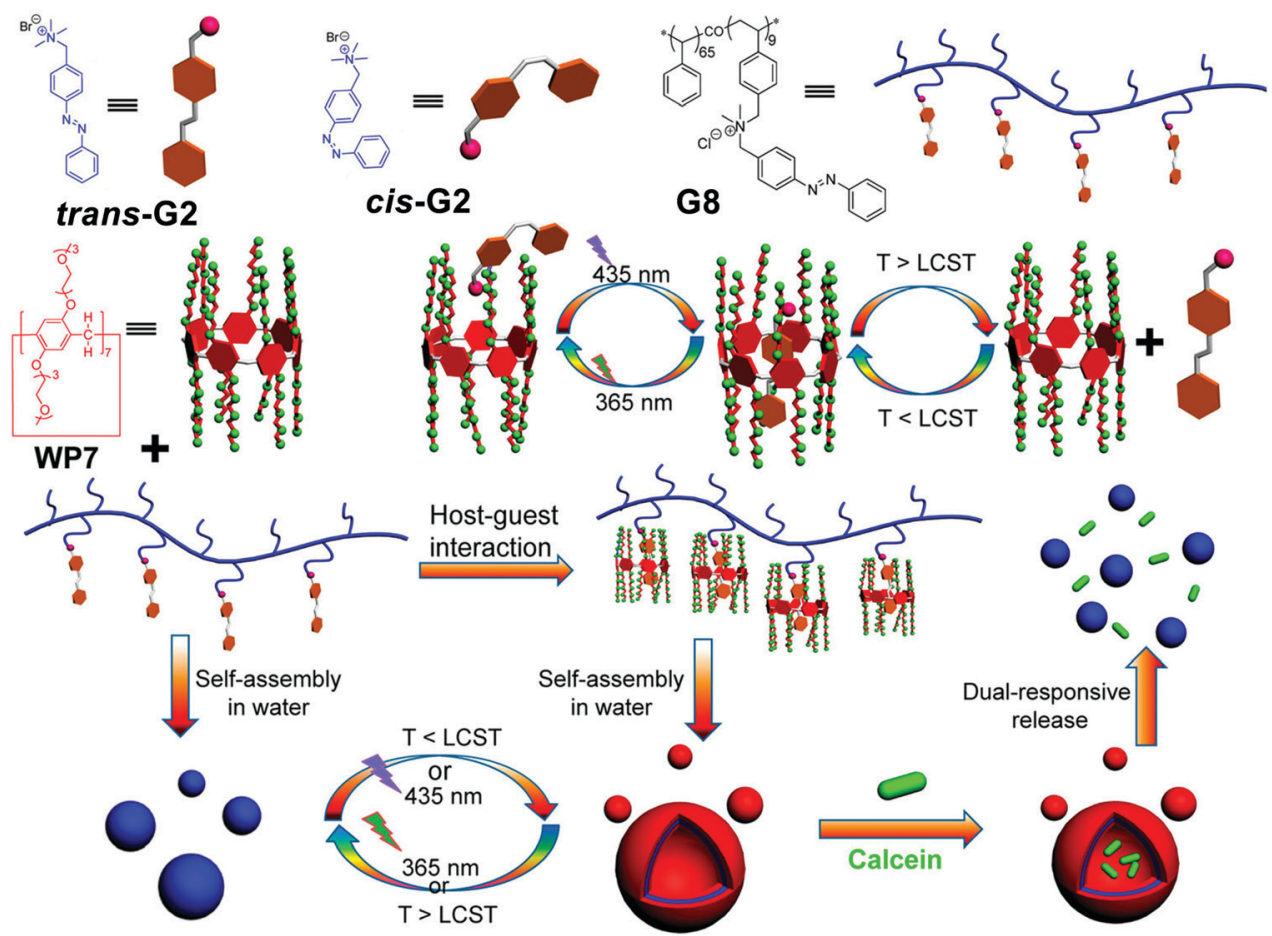

Fig. 7 Chemical structures of WP7, G2, and polymer G8, and cartoon representation of the controlled release process. Reproduced with permission from ref. 74. Copyright 2015, American Chemical Society.

\section{WP[n]-based nanocarriers for drug/gene/protein delivery}

\subsection{WP $[n]$-based nanocarriers for drug delivery}

In recent years, many scientists have paid their attention to the development of supramolecular DDS based on $\mathrm{WP}[n] \mathrm{s} .^{79,80}$ In this section, we will discuss various DDS based on $\operatorname{WP}[n]$ nanocarriers, which were constructed from different types of host-guest building blocks, such as tadpole-type, bola-type, prodrug-type, and polymeric supra-amphiphiles.

Previously, Wang and co-workers found that ferrocene can well complex with pillar[6]arene in organic solvents. ${ }^{81}$ In 2013 , they further reported a supramolecular nanocarrier constructed from a tadpole-type supra-amphiphile based on WP6a and a ferrocene-based guest (Fc) (Fig. 8a). ${ }^{82}$ The Fc group on the guest can bind with WP6a while the long alkyl chain on the guest provides a hydrophobic part. The resultant tadpole-type supraamphiphile could further assemble into vesicles in aqueous media. Mitoxantrone (MTZ) is a hydrophilic anticancer drug, which was employed as a model in this work to evaluate the delivery efficiency of this system. The release of the drug could be controlled by changing the $\mathrm{pH}$ of the solution. The release rate was slow under neutral $\mathrm{pH}$ conditions. However, the drug was released rapidly in an acidic environment. Since the microdomain of cancer cells is acidic, the rapid release of MTZ from the nanocarriers is very important for targeted therapy. Subsequently, cytotoxicity experiments were conducted (Fig. 8b and c). The cytotoxicity measurements of the system against normal cells (NIH3T3 cells) showed that the blank nanocarriers had low toxicity. By loading MTZ in the nanocarriers, the toxicity of the drug could be alleviated against NIH3T3 cell lines. Moreover, the cellular uptake experiments showed that the drugloaded nanocarriers could easily go inside SMMC-7721 cancer cells. The encapsulation of MTZ by such nanocarriers did not influence the therapeutic efficacy of the drug. This study pioneered the employment of $\mathrm{WP}[n]$-based nanocarriers for DDS.

In another work, Wang and co-workers constructed a multistimuli-responsive vesicle from WP6a and a pyridinium derivative guest. ${ }^{83}$ Drug loading and controlled release tests showed that the anticancer drug DOX was well loaded into the nanocarriers, and the obtained vesicles displayed good $\mathrm{Ca}^{2+}$ - and pH-responsiveness. Moreover, cellular uptake experiments displayed that the encapsulation of the drug into the nanocarriers did not reduce the therapeutic efficacy of the drug against tumor cells. By contrast, the cytotoxicity of DOX for normal cells was significantly decreased. This case provided a strategy for the construction of smart nanocarriers, which showed great potential as a novel DDS. Later, they constructed another example of a stimuli-responsive drug nanocarrier with dual responsiveness toward light and $\mathrm{pH}^{84}$ The $\mathrm{pH}$ responsiveness originates from the host WP6a, while the photo-responsiveness comes from the azobenzene group of the guest. The obtained nanocarriers could encapsulate MTZ, which showed both $\mathrm{pH}$ - and UV-responsive release. This nanocarrier system with dual-stimuli responsiveness displayed great potential as a smart DDS.

The disulfide bond is well known to be labile to GSH that is generally overproduced in cancer cells. In 2015, Hu, Wang and 

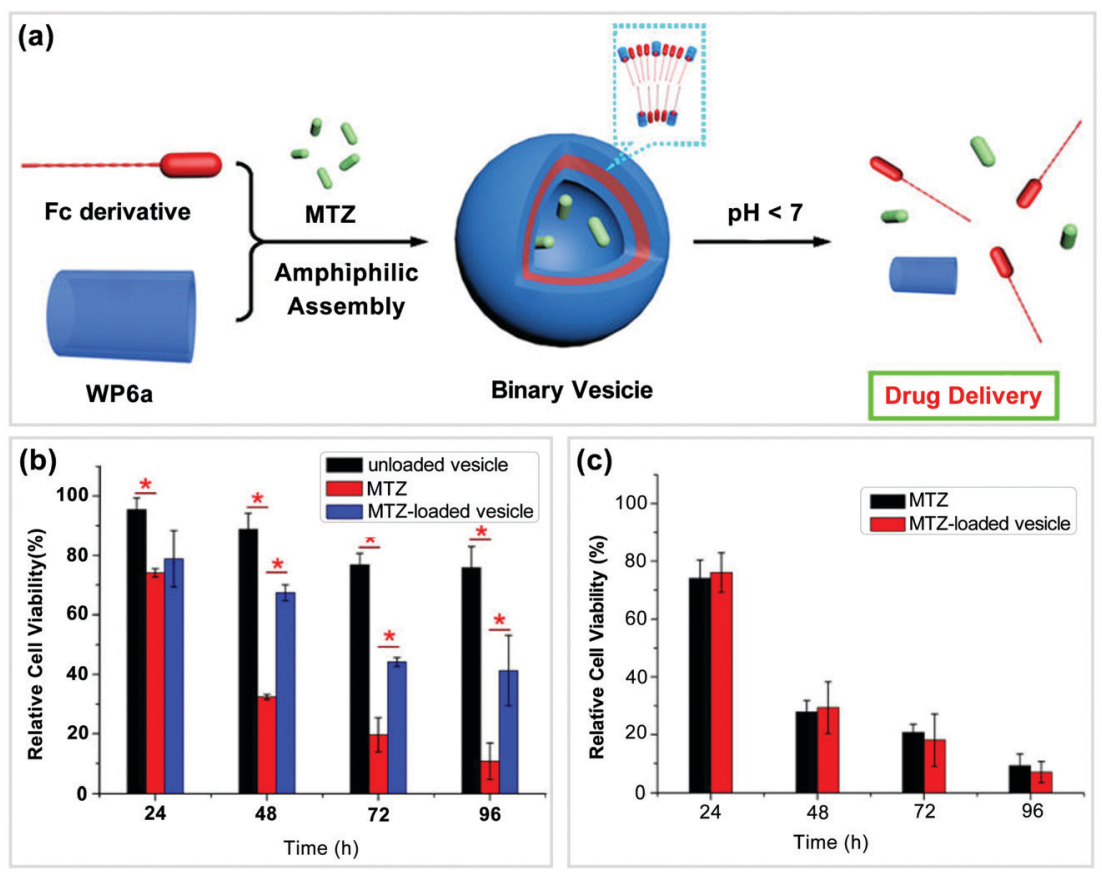

Fig. 8 (a) Cartoon pictures of supramolecular vesicles based on WP6a/Fc and their drug release process, (b) effect of different vesicles on the viability of NIH3T3 cells, and (c) anticancer activity of different vesicles against SMMC-7721 cancer cells. Reproduced with permission from ref. 82. Copyright 2013, American Chemical Society.

co-workers prepared a dual $\mathrm{pH}$ - and GSH-sensitive nanocarrier constructed from WP5a and a guest containing a disulfide bond. ${ }^{85}$ The host and the guest could be complexed through the lysine group in the guest molecule. This type of nanocarrier can also encapsulate MTZ and release it rapidly in the presence of GSH. HepG2 cancer cells were employed to evaluate the anticancer efficacy of this system. The results showed that the cell viability was only about $10 \%$ after $72 \mathrm{~h}$ of incubation of cancer cells with MTZ-loaded vesicles, exhibiting better therapeutic effects than free MTZ (26\%). In 2016, they further fabricated biocompatible nanocarriers from phosphate $\mathrm{WP}[n] \mathrm{s}$ and a pyridinium bromide guest for anti-cancer therapy. ${ }^{86}$ The introduction of phosphate groups was aimed to enhance the water-solubility and biocompatibility of WP $[n]$. Micelles were formed via self-assembly from WP5c and the guest, while hollow vesicles were formed from WP6c and the guest. Both of them exhibited dual $\mathrm{pH}-$ and $\mathrm{Zn}^{2+}$ responsiveness. DOX was loaded inside the micelles while MTZ could be encapsulated into the resultant vesicles. This study provided a novel method for the construction of smart drug delivery nanocarriers with different morphologies.

As shown in Fig. 1, a bola-type amphiphile bears two hydrophilic terminals and a hydrophobic middle part. Generally, bola-type amphiphiles show a lower critical aggregation concentration (CAC) and a higher structural stability than tadpole-type supra-amphiphiles. In 2014, Huang and co-workers prepared a bola-type supra-amphiphile based on WP5b and a rod-coil guest. ${ }^{87}$ This supra-amphiphile could act as a $\mathrm{pH}$ and paraquat sensor. In 2016, Shi and co-workers prepared a dual-stimuli responsive nanocarrier from a bola-type supra-amphiphile based on WP6b and a photo-responsive guest. ${ }^{88}$ The guest is an azobenzene derivative which could be aggregated into nanosheets.
The complexation of the guest and WP6b resulted in the formation of a supra-amphiphile, which can further self-assemble into vesicles. The obtained nanocarriers displayed both photoand pH-responsiveness. Controlled release of DOX by such a system was successfully realized.

Another excellent example of DDS constructed from bola-type supra-amphiphiles was reported by Zhang, $\mathrm{Hu}$ and co-workers. ${ }^{89}$ This dual $\mathrm{pH}$ - and GSH-responsive nanocarrier was realized by the recognition of G9 by WP5a (Fig. 9). Guest G9 is a bola-type naphthalimide compound. An efficient nanocarrier should be responsive to stimuli present in the tumor microenvironment to release the loaded drug. In this case, the anticancer drug DOX was efficiently loaded and released by the obtained nanocarrier. The fluorescence spectra showed that no leakage of DOX was observed under both neutral $\mathrm{pH}$ and GSH-free conditions. Notably, DOX release was detected clearly in the presence of GSH. Biocompatibility experiments showed that this bola-type drug nanocarrier had good biocompatibility by using MRC-5 cells. Cellular uptake experiments showed that these nanocarriers entered tumor cells mostly by endocytosis. This research offers an alternative method to develop smart nanocarriers with dual stimuli-responsiveness for specific drug delivery.

Nanocarriers constructed from polymeric supra-amphiphiles are an alternative good choice for DDSs. In 2017, Hu, Zhu, Wang and co-workers employed an efficient approach for fabricating nanocarriers by host-induced polymer assembly (Fig. 10). ${ }^{90}$ In this example, polyglutamamide with butyl-ammonium (BA) groups was used as a polymer guest. Furthermore, the polymeric guest was equipped with a targeting biotin unit. The BA groupcontaining polymer 1 could form supramolecular polymersomes in the presence of WP5a. MTZ was employed as a model 


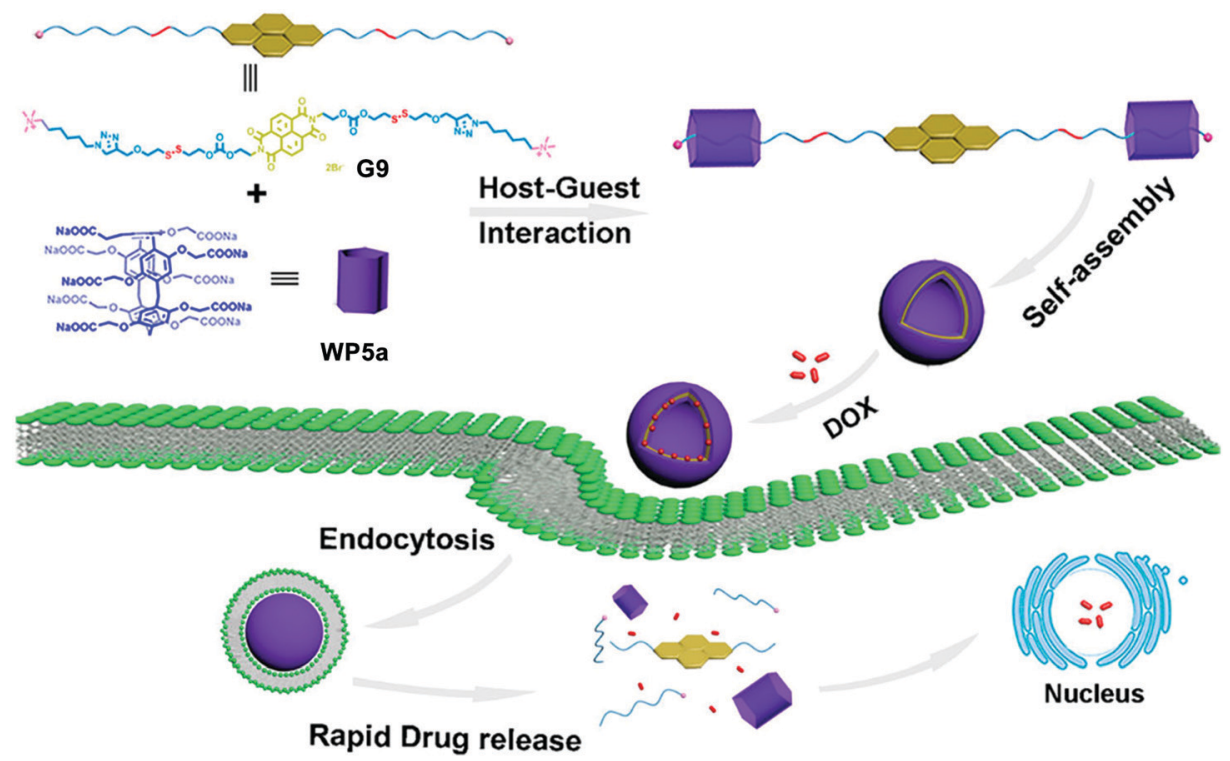

Fig. 9 Schematic illustration of fabricating dual-stimuli responsive nanocarriers from bola-type supra-amphiphiles based on WP5a and G9 and their application in drug release. Reproduced with permission from ref. 89. Copyright 2017, American Chemical Society.

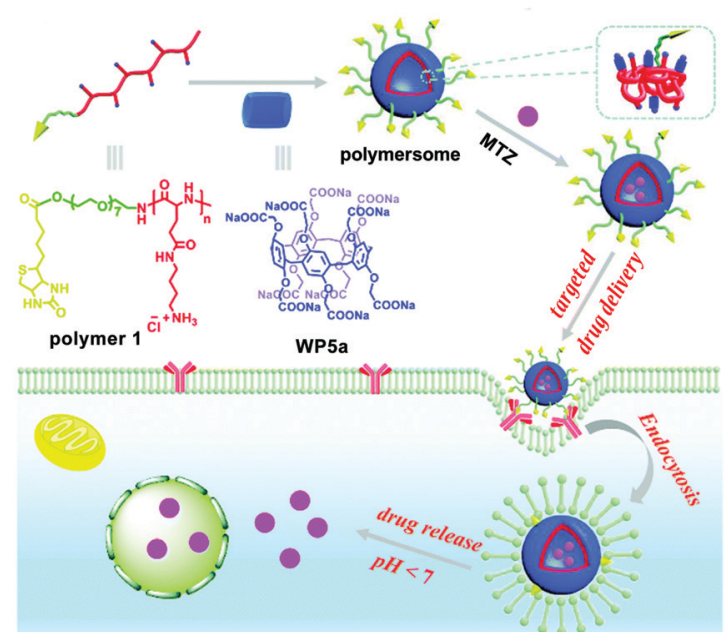

Fig. 10 Schematic representation of the fabrication of polymersomes and their applications in targeted drug delivery. Reproduced with permission from ref. 90. Copyright 2017, the Royal Society of Chemistry.

drug to investigate the loading efficiency and $\mathrm{pH}$ stimulated drug release of such nanocarriers. UV-vis spectra showed that the drug loading efficiency was up to $52 \%$. Moreover, the MTZ release percentage within $12 \mathrm{~h}$ was calculated to be $79 \%$ when the $\mathrm{pH}$ value was adjusted to 5.2. NIH3T3 cells (normal cells) were incubated with the drug to evaluate the cytocompatibility of these nanocarriers. The terminal of polymer 1 was attached to the biotin ligand, endowing the nanocarrier with a targeting capability. Therefore, the nanovehicles could deliver MTZ to biotin receptor-positive HeLa cells. This strategy not only decreased the cytotoxicity of MTZ to normal cells but also strengthened its anticancer efficacy. This research offered a new method to fabricate functional nanocarriers, which may have potential applications in biomedical fields.
Supramolecular brush copolymers (SBPs) constructed by non-covalent interactions have also exhibited tremendous advantages. For example, Chen, Huang and co-workers reported an amphiphilic SBP (P5-PEG-Biotin $\supset$ PTPE) based on a WP[5] (P5) and a 4,4'-bipyridinium derivative (M) (Fig. 11). ${ }^{91}$ P5-PEG-Biotin $\supset$ PTPE can form supramolecular NPs (SNPs), which could serve as a self-imaging nanocarrier because of the aggregation-induced emission (AIE) effect of tetraphenylethene (TPE) groups. The core-shell type SNPs were used as a nanovehicle to encapsulate DOX. In the SNPs, the TPE motifs served as donors, while the anticancer drug DOX groups played the role of acceptors. The association constant of the model host $\mathbf{P 5}$ and the model guest $\mathbf{M}$ was measured to be $(2.50 \pm 0.16) \times 10^{4} \mathrm{M}^{-1}$ by utilizing fluorescence titrations. Subsequently, the amphiphilic SBP (P5-PEG-Biotin $\supset$ PTPE) was fabricated, in which the backbone of TPE served as the hydrophobic sections and the PEG chains acted as the hydrophilic parts. Flow cytometry investigations on HeLa and HEK293 cells proved that the biotin units linked on the DOX-loaded SNPs remarkably enhanced targeted cellular uptake of the nanocarriers. Finally, HeLa tumor bearing Balb/c mice were injected with free DOX or drug-loaded SNPs to evaluate drug accumulation and distribution in vivo. The results showed that in most organs the uptake of DOX-loaded SNPs was less than free DOX, suggesting that the non-specific toxicity may get reduced by employing SNPs as drug delivery nanocarriers. This work offered a new supramolecular strategy for the fabrication of self-imaging DDSs, which may hold significant potential in nanotherapy. In another similar study, the same group of researchers constructed a supramolecular diblock copolymeric amphiphile by using the same host P5-PEG-Biotin and a polymeric guest based on a viologen salt. ${ }^{92}$ This host-guest complex formed vesicular nanocarriers that were also successfully used as drug delivery carriers for DOX.

In order to enhance the drug loading efficiency and promote controlled drug release of nanocarriers, a prodrug has emerged 

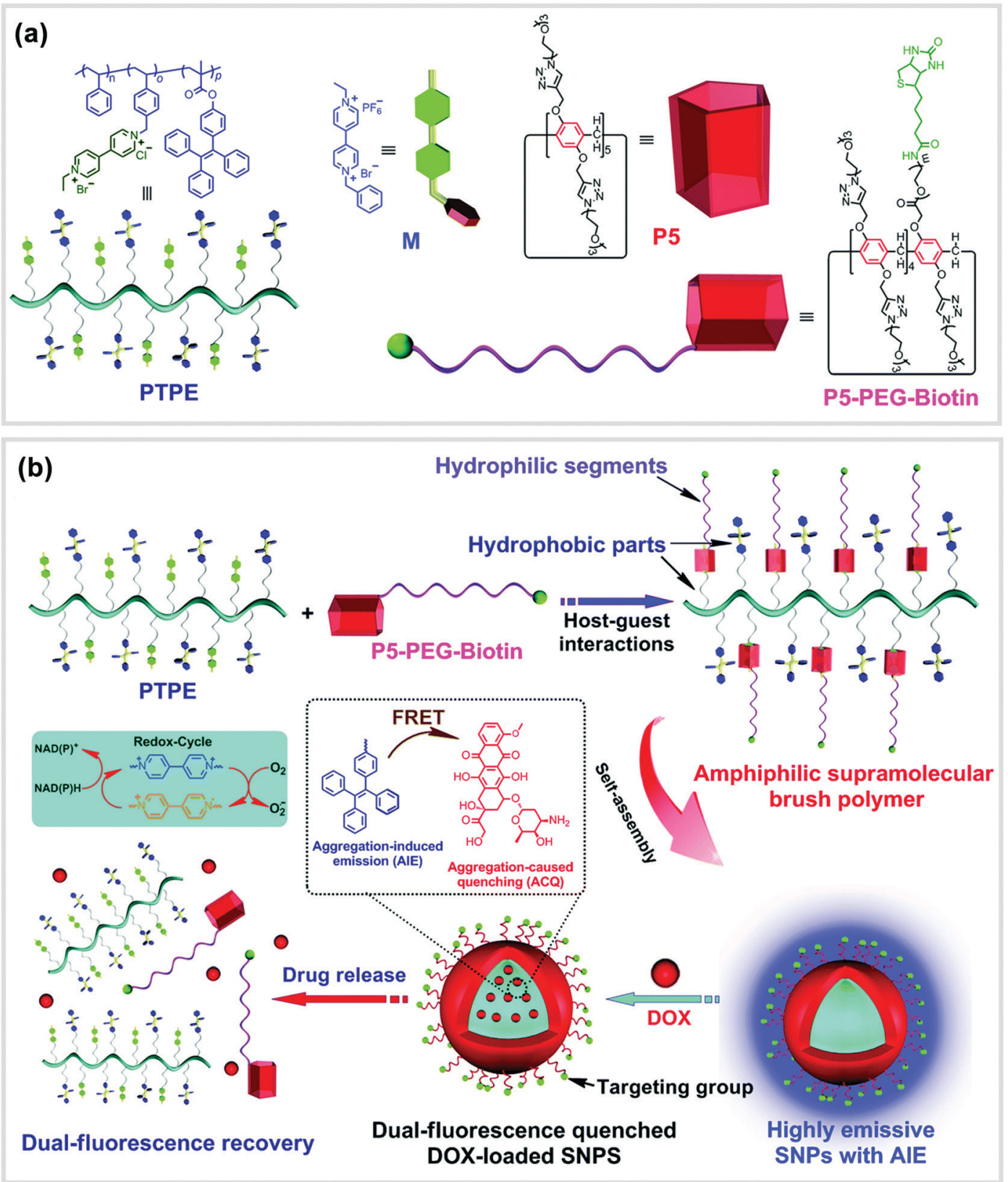

Fig. 11 (a) Chemical structures of PTPE, M, P5 and P5-PEG-Biotin. (b) Cartoon representation of the formation of SNPs and their application as nanocarriers. Reproduced with permission from ref. 91. Copyright 2016, the Royal Society of Chemistry.

as a promising strategy. For the prodrug strategy, a parent drug is generally bound to functional groups and the entire species can act as a guest structure. The prodrug should be inactive in the normal state but can be metabolized or degraded into the parent drug upon environmental stimulation. For this purpose, $\mathrm{Hu}$, Wang and co-workers constructed NPs self-assembled from supra-amphiphiles based on WP6a and a DOX-based prodrug guest (Fig. 12). ${ }^{93}$ The DOX-based guests (G10 and G11) were prepared by connecting an anticancer drug DOX unit with a pyridinium group through an acid-cleavable hydrazone bond. The release of the drug was less than $12 \%$ at $\mathrm{pH} 7.4$. However, a rapid release of the drug could be achieved at $\mathrm{pH}=5.5$ owing to the hydrolysis of hydrazone bonds. Meanwhile, the cumulative release was almost up to $100 \%$ in half an hour. Further studies showed that WP6a could accelerate the hydrolysis of hydrazone bonds due to an intramolecular catalysis process. Compared with free DOX, the obtained NPs showed reduced toxicity. The cellular internalization of these nanocarriers and free DOX was tested by using SKOV3 cells. The results showed that these DOX-conjugated nanocarriers had the ability to deliver DOX in an efficient manner. This study provided a novel design strategy for constructing a DDS based on a prodrug. Similar to this example, a supramolecular prodrug based micelle constructed from WP5b and methyl viologen functionalized DOX was also reported by Ji and co-workers. ${ }^{94}$ The nanocarriers were aggregated under an acidic environment, resulting in promoted drug accumulation and treatment efficacy. 


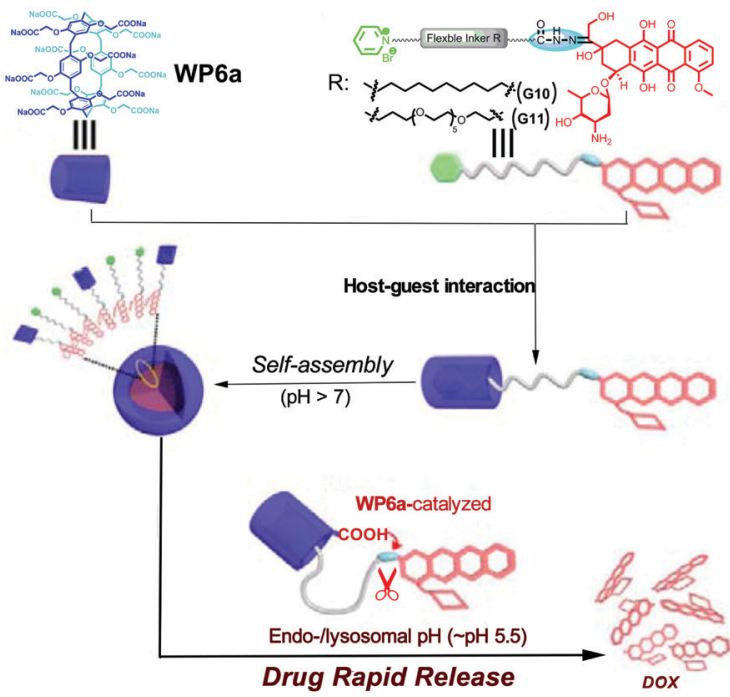

Fig. 12 Cartoon representation of the self-assembly of prodrug NPs based on WP6a and G10 or G11. Reproduced with permission from ref. 93. Copyright 2015, American Chemical Society.

Combination of two anticancer drugs for cancer treatment can remarkably overcome the drug resistance of tumor cells. Recently,
$\mathrm{Hu}$ and co-workers reported the preparation of a drug-drug conjugate supramolecular nanocarrier based on WP6a. ${ }^{95}$ In this study, two anticancer drugs, chlorambucil and camptothecin, were linked by a disulfide bond to achieve a new conjugated prodrug guest, which complexed with WP6a via host-guest recognition. The complex further self-assembled into vesicles in aqueous media. GSH-responsive disulfide bonds can be destroyed inside tumor cells. As a result, both of the drugs could be released simultaneously to realize the synergistic therapy against cancer cells. Moreover, cytotoxicity measurements confirmed that the obtained nanocarriers not only killed tumor cells but also remarkably reduced the side effects against normal cells. This work provided an interesting method for the fabrication of nanocarriers with great potential for synergistic chemotherapy.

In a follow-up study, $\mathrm{Hu}$, Schmuck and co-workers fabricated tumor-targeting nanocarriers by using a WP[5]-derived prodrug and a functional guest. ${ }^{96}$ The host is a WP[5]-based prodrug (WP5-DOX) which also possesses a hydrazone linkage (Fig. 13). The guest is an Arg-Gly-Asp (RGD)-modified sulfonate molecule (RGD-SG) which has both the targeting capability realized by RGD and host-binding ability achieved by sulfonate groups. Therefore, these nanocarriers can carry DOX to RGD receptorpositive tumors. Moreover, the structures of the nanocarriers

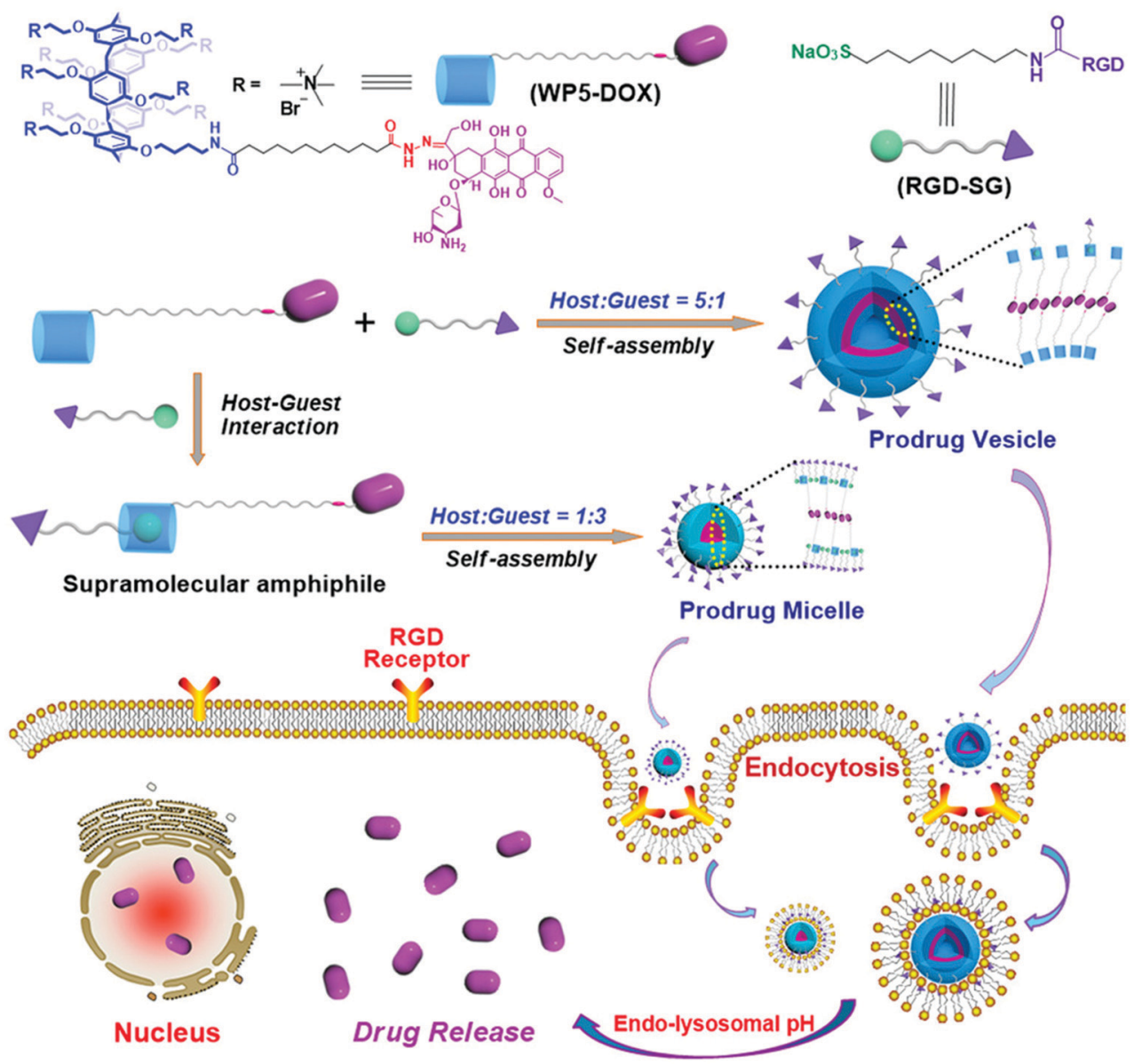

Fig. 13 Schematic illustration of the fabrication of targeting supramolecular prodrug nanocarriers and their applications for targeted drug delivery. Reproduced with permission from ref. 96. Copyright 2018, Wiley Publishers. 
were tuned between micelles and vesicles by adjusting the molar ratio of the host and the guest. Cytocompatibility experiments showed that the DOX loaded nanocarriers had less cytotoxicity against 293T and WI-38 normal cell lines than free DOX, indicating a good cytocompatibility of these nanocarriers. These drug loading nanocarriers also exhibited longer blood circulation time, enhanced anticancer efficacy, and alleviated systemic cytotoxicity in a murine tumor model, suggesting a significant potential for targeted drug delivery to treat cancer.

Galactose derivatives show high affinity for galectin overexpressing hepatoma tumor cells. Hence, the introduction of the galactose group onto nanocarriers is an efficient strategy to develop a targeting DDS. To this end, Pei and co-workers developed novel nanocarriers from tryptophan-modified $\mathrm{WP}[5]$ and a galactose-derived guest. ${ }^{97}$ The galactose group on the surface of the self-assembled vesicles served as a targeting moiety to an asialoglycoprotein receptor (ASGP-R) overexpressed by HepG2 cells. The indole rings of tryptophan in the nanocarriers can interact with DNA, which synergistically enhanced their cytotoxicity toward cancer cells. In a subsequent study, Pei and co-workers fabricated multifunctional nanocarriers based on the complexation of ferrocenecarboxylic acid capped $\mathrm{WP}[5]$ and the same guest discussed in the previous case for targeted DOX delivery. ${ }^{98}$ In 2018, they further constructed a hybrid nanomaterial on the basis of the host-guest recognition between WP6b and the above mentioned galactose guest, as well as the DOX-loaded zeolitic imidazolate framework (ZIF-8) for targeted drug delivery. ${ }^{99}$

\subsection{WP $[n]$-based nanocarriers for gene delivery}

Nowadays, drug resistance is a major problem in chemotherapy. A possible solution to this problem is co-delivery of a drug and a small interfering RNA (siRNA). Such a system should ideally hold the capability to respond to the specific changes in the microenvironment of cancer cells, leading to the release of the drug and siRNA concurrently. As a result, siRNA could silence drug resistance related genes. According to this strategy, Pei and co-workers constructed a ferrocenium-capped pillar[5] arene (FCP), which was oxidized to amphiphilic WP[5] (FCAP), which was further self-assembled into cationic vesicles for drug/siRNA co-delivery (Fig. 14). ${ }^{100}$ First, HeLa cells and 293T cells were used to investigate the anticancer efficacy and safety profile in vitro. 293T cells (normal cells) showed higher viabilities upon incubation with drug loaded nanocarriers than those with free DOX. In contrast, HeLa cells displayed significantly decreased viabilities when incubated with drug loaded nanocarriers, when compared with those incubated with free DOX. Subsequently, they chose drug resistance gene silencing siRNA (MRP1 siRNA) and DOX as a siRNA/drug couple for co-delivery research against human ovarian SKOV-3 cancer. The results showed that the DOX resistance was overcome after the tumor cells were transfected with MRP1 siRNA. As a consequence, the therapeutic efficacy of chemotherapy was greatly enhanced by the combination of DOX and MRP1 siRNA. Therefore, this study offered an excellent example of a drug/siRNA co-delivery platform based on $\mathrm{WP}[n]$ for improved anticancer therapy.

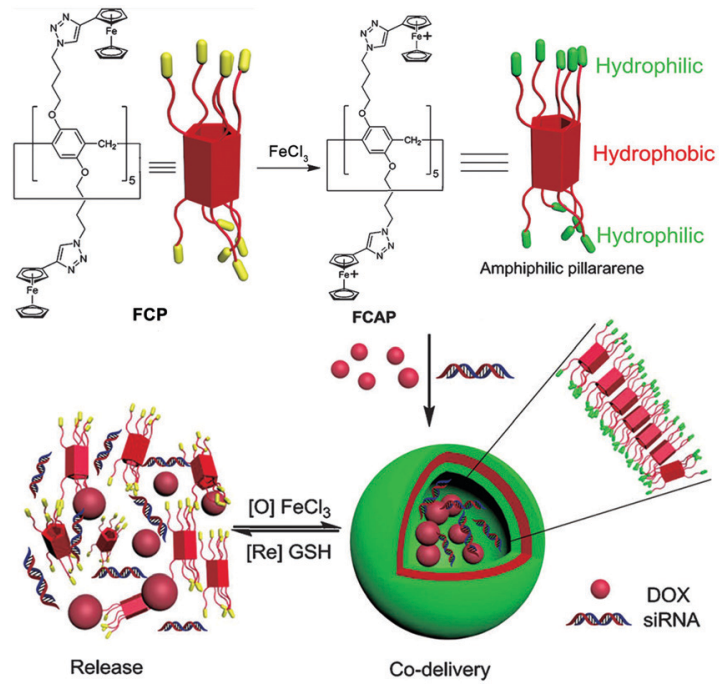

Fig. 14 Cartoon representation of the co-delivery of drug/siRNA in a ferrocenium-capped WP[5]-based nanocarrier. Reproduced with permission from ref. 100. Copyright 2014, Wiley Publishers.

\subsection{WP $[n]$-based nanocarriers for protein delivery}

An increase in the glucose level of plasma may be indicative of diabetes, which is caused by insufficient secretion of insulin from the pancreas. Therefore, the development of nanocarriers with glucose-responsiveness may be employed for controlled delivery of insulin. Recently, Hu, Wang and co-workers fabricated smart nanocarriers responsive to glucose by employing WP5a and a guest containing a pyridylboronic group. ${ }^{101}$ The cargo loading and release experiments proved that insulin could be loaded into the nanocarriers efficiently (64\%), indicating that supramolecular vesicles can indeed encapsulate large protein molecules. Moreover, the cargo loaded vesicles exhibited rapid release of insulin in response to a relatively high concentration of glucose. Cytotoxicity experiments suggested that these nanocarriers displayed good cytocompatibility suitable for insulin delivery. This work opened a new window for the fabrication of glucose-responsive nanocarriers, which can be used for diabetes treatment.

In another work, Hu, Zhang, Jiang, Wang and co-workers further developed glucose-responsive nanocarriers (Fig. 15). ${ }^{102}$ In this work, a diphenylboronic acid derivative (G12) bearing a pyrene unit and a quaternary ammonium group was designed and synthesized, which could complex with WP5a to selfassemble into nanocarriers. Herein, the diphenylboronic acid moiety in G12 can specifically recognize glucose to form a 1:1 binding complex. WP5a improved the water solubility of G12 and induced self-assembly of the supra-amphiphile. Meanwhile, the $\pi-\pi$ interactions between pyrene units produced long-wavelength excimer emission, making glucose monitoring possible by the naked eye. This system could carry insulin together with glucose oxidase (GOx), which can oxidize glucose to gluconic acid, resulting in the production of $\mathrm{H}_{2} \mathrm{O}_{2}$ and a decrease of local $\mathrm{pH}$. As a result, this process promoted rapid disassembly of the nanocarriers due to the protonation of WP5a and cleavage of 


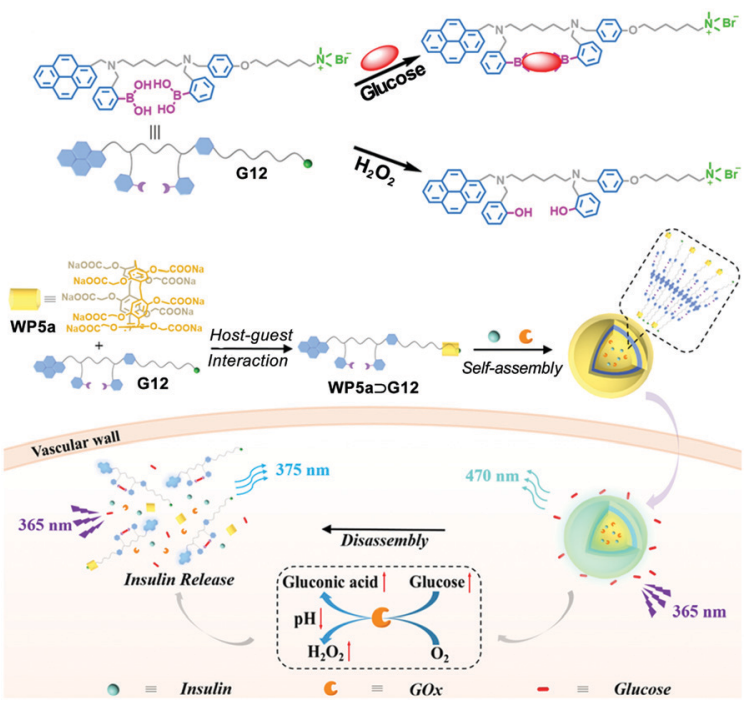

Fig. 15 Schematic illustration of the insulin delivery nanocarriers based on WP5a and G12 with glucose-responsiveness. Reproduced with permission from ref. 102. Copyright 2018, Wiley Publishers.

C-B bonds by $\mathrm{H}_{2} \mathrm{O}_{2}$, achieving an efficient insulin release. More interestingly, in vivo studies by employing a mouse model of type I diabetes demonstrated that the insulin and GOx loaded nanocarriers could maintain the blood glucose level at a normal level for a long period of time. Notably, this work pioneered a theranostic system for glucose-sensing by employing a simple artificial nanocarrier, which can achieve continuous glucose monitoring and closed-loop diabetes treatment at the same time.

\section{WP[n]-based nanocarriers for photodynamic/photothermal therapy}

Besides chemotherapy, photodynamic therapy (PDT) and photothermal therapy (PTT) are important alternative choices for cancer treatment. ${ }^{103-107}$ PDT and PTT can sometimes overcome some of the difficulties that chemotherapy can't solve. Therefore, a variety of nanocarriers based PDT and PTT systems have been developed as a promising strategy for cancer treatment in recent years. PDT therapy is generally based on a photosensitizer that can generate reactive oxygen species (ROS) upon photo irradiation, resulting in cytotoxicity against tumor cells. PTT is based on cytotoxic heat generated by photothermal agents upon light irradiation to ablate cancer cells. Therefore, both PDT and PTT are non-invasive therapeutic modalities. Furthermore, dual-modality therapy, for example, chemo-photodynamic therapy or chemo-thermodynamic therapy, and even multi-modality therapy, has also attracted increasing interest, because combination therapy can often overcome drawbacks faced by single-modality treatment.

\subsection{WP $[n]$-based nanocarriers for photodynamic therapy}

Mitochondria are an important class of subcellular organelle, which hava a key role in cellular metabolism. Combination of mitochondria-targeting agents with photodynamic therapy is a promising strategy to selectively kill cancer cells. Under this principle, Zhang and co-workers constructed a system by employing supra-amphiphiles based on WP5a and a tetraphenylporphyrin derivative (the guest) as a photosensitizer to realize PDT. ${ }^{108}$ The guest itself exhibited no self-aggregation behavior in water. In the presence of WP5a, the guest complexed with the host and further formed NPs. The supramolecular NPs showed silent fluorescence and PDT activity under physiological conditions, and subsequently could efficiently release and activate the photosensitizer in an acidic environment. The photosensitizer produced ${ }^{1} \mathrm{O}_{2}$ upon light irradiation, which damaged the mitochondria and finally caused the death of tumor cells. In another work by the same group of researchers, they fabricated a reduction-responsive porphyrin photosensitizer delivery system by employing supra-amphiphiles based on a poly(ethylene glycol)-derived WP[5] and a porphyrin derivative containing a disulfide bond. ${ }^{109}$

Peptide aggregates with multiple nanoarchitectures have potential applications in advanced biomaterials. However, the tedious and costly covalent peptide modification impedes the exploration of their application. Very recently, Yu, Mao, Huang and co-workers developed a simple strategy to fabricate a supramolecular peptide based on WP[5], which can be used for PDT (Fig. 16). ${ }^{110}$ The guest molecule (PyP) contains a peptide sequence (G7CCERGDS), a hydrophobic alkyl chain, and a 4-methylpyridinium group that could bind with WP[5]. Moreover, the peptide sequence encompasses a targeting heading group (ERGDS) and a cross-linker precursor (cysteine units). WP[5] (WP5e) with LCST behavior was employed as the host. The guest molecule without the pyridinium group (PA) was self-assembled into nanofibers upon the addition of $\mathrm{Ca}^{2+}$. When PyP complexed with WP5e, irregular sheet-like aggregates with different sizes appeared. Interestingly, the sheet-like assemblies transformed into NPs when the temperature was increased to $45{ }^{\circ} \mathrm{C}$. At this temperature, WP5e became hydrophobic, leading to the formation of a supra-amphiphile, which further self-assembled into NPs. Meanwhile, the cysteine units on the guest could be oxidized to disulfide upon heating in air, resulting in cross-linked shells that prevented the disruption of the nanocarriers during the cooling process. Finally, tetrakis(4-hydroxyphenyl)porphyrin (TPP) was successfully used as a photosensitizer which could be encapsulated into the NPs for PDT. In contrast to free TPP, TPP@NPs displayed enhanced internalization and PDT efficacy against A549 cells. In vivo studies demonstrated that the resultant nanocarriers had high anti-tumor efficacy towards 4T1 tumors. This study provided an attractive strategy to construct supramolecular peptides based on WP[5] using a controllable self-assembly process, which holds great potential in precise anticancer therapy.

\subsection{WP $[n]$-based nanocarriers for chemo-photodynamic or chemo-photothermal therapy}

In order to take advantage of combination therapy, $\mathrm{Hu}$, Wang and co-workers synthesized a boron-dipyrromethene derived compound (G13) which played the roles of both a guest and a photosensitizer (Fig. 17). ${ }^{111}$ Molecule G13 can complex with WP5a in aqueous media to form a supra-amphiphile, which can further self-assemble into supramolecular vesicles. The obtained nanocarriers had high DOX encapsulation efficiency. Moreover, 
a
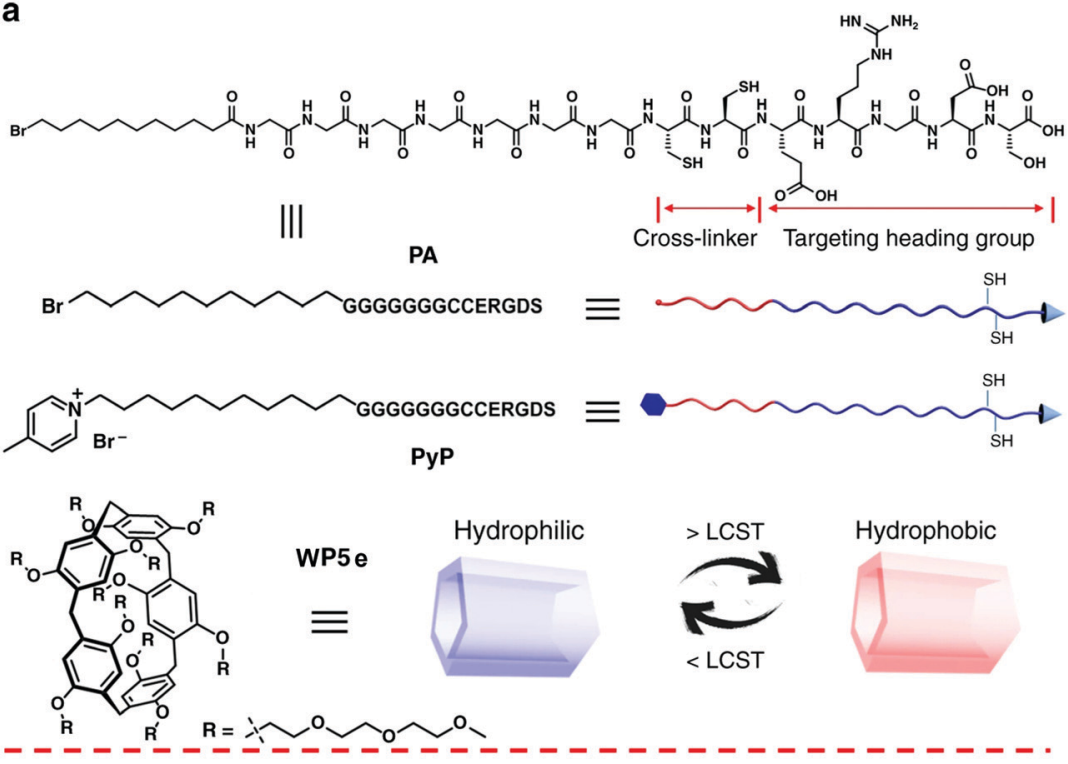

b
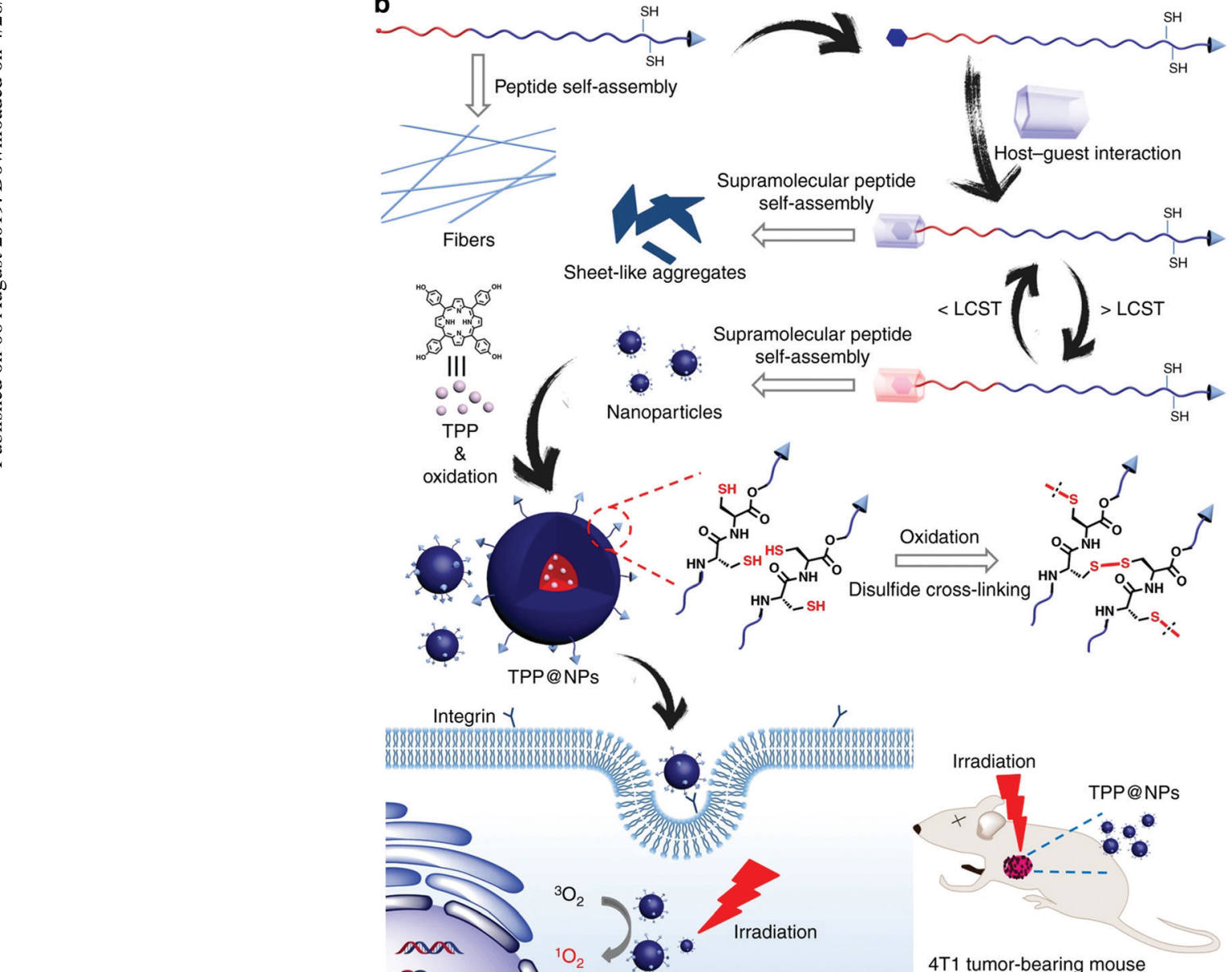

Fig. 16 (a) Cartoon representations of PA, PyP, and WP5e. (b) Schematic illustrations of the programmable peptide self-assembly and PDT processes. Reproduced with permission from ref. 110. Copyright 2019, Nature Publishing Group.

the loaded nanocarriers exhibited rapid drug release under acidic conditions. Cytotoxicity experiments demonstrated that such vesicles had good biocompatibility. Cellular internalization tests suggested that the loaded nanocarriers could be well localized in lysosomes. Finally, the obtained DOX-loaded nanocarriers further showed significant therapeutic efficacy in 


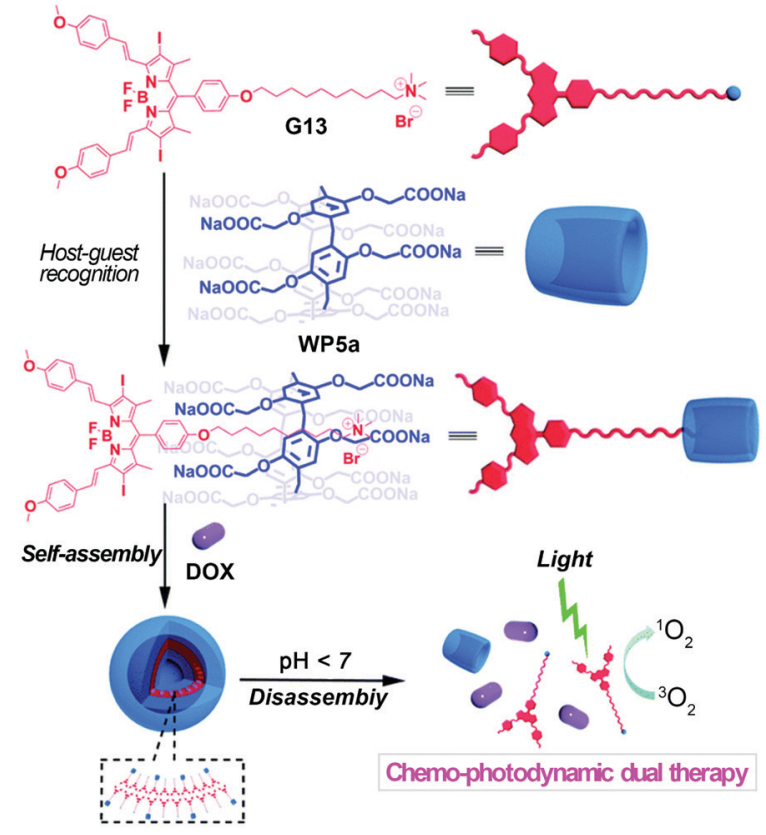

Fig. 17 Cartoon representation of the construction of nanocarriers based on WP5a and G13 and their applications for chemo-photodynamic therapy. Reproduced with permission from ref. 111. Copyright 2015, the Royal Society of Chemistry.

combination with photodynamic and chemo-activities towards A549 cancer cells, indicating promising applications for dual chemo-photodynamic treatment.

CuS NPs have been broadly investigated as PTT agents due to their low cytotoxicity, facile preparation, and high photothermal conversion efficiency. ${ }^{112}$ In 2018 , Yu and co-workers constructed a nanoplatform that integrated photothermal therapy, chemotherapy, and targeting functions together (Fig. 18). ${ }^{113}$ This nanocarrier system was based on WP5a-functionalized CuS NPs (CuS@WP NPs), which showed good monodispersibility and strong NIR absorption in water. The CuS@WP NPs were further tethered with a liver cancer-targeting galactose derivative G14 via host-guest recognition to form CuS@WPG NPs. The obtained nanocarriers showed good photothermal ablation ability toward

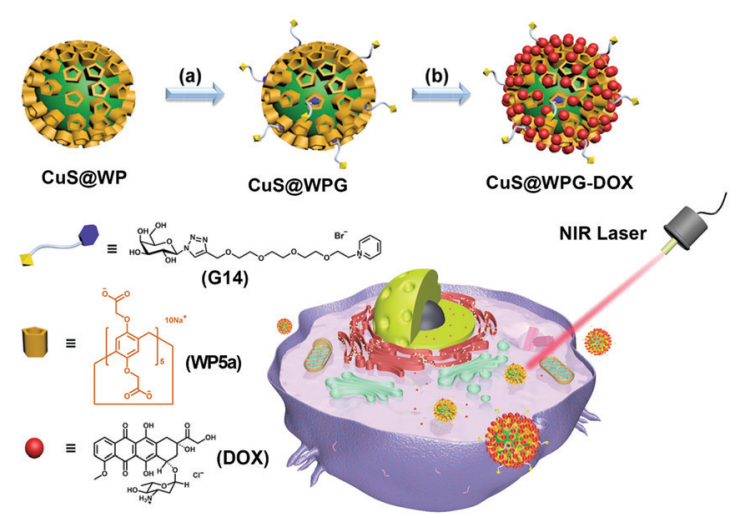

Fig. 18 Cartoon representation of the construction of CuS@WPG-DOX and its drug-release process. Reproduced with permission from ref. 113. Copyright 2018, American Chemical Society.
HepG2 cells under light at $808 \mathrm{~nm}$. Furthermore, DOX could be loaded on CuS@WPG via electrostatic interactions. The drug-loaded nanocarriers also displayed excellent, controlled drug release in an acidic environment. Interestingly, this supramolecular nanocarrier exhibited a significantly enhanced therapy efficacy via the combined targeting and chemophotothermal therapy in vitro study. Finally, they performed preliminary in vivo studies which proved that such NPs had excellent cytocompatibility and good cancer inhibition capability upon NIR laser irradiation. This study offered a useful method to fabricate nanosystems for potential biomedical applications by employing organic-inorganic hybrid materials.

In 2018, Fan and co-workers constructed supramolecular vesicles for combined chemo-photothermal therapy. ${ }^{114}$ They synthesized a perylene diimide dye derivative which not only played the role of a photothermal agent, but also acted as a guest. The guest could complex with WP5a to form supramolecular vesicles, which exhibited high-loading capacity for hydrophobic DOX. Notably, cytotoxicity experiments showed that synergistic effects of the nanocarriers against MCF-7 cancer cells were realized, exhibiting much better efficacy than PTT treatment or chemotherapy alone. Moreover, these drug nanocarriers could easily enter tumor cells via endocytosis to achieve good intracellular accumulation of anticancer drugs. This study also offered a new approach to construct nanocarriers for synergistic antitumor therapy.

\subsection{WP $[n]$-based nanocarriers for combined chemo/ photodynamic/photothermal therapy}

The combination of multiple therapeutic modalities may produce more efficient treatment and minimize side effects. Very recently, Fan and co-workers continued to construct multifunctional supramolecular vesicles assembled from WP5a and a bola-type NIRabsorbing guest G15 (Fig. 19). ${ }^{115}$ These supramolecular vesicles showed significant anticancer efficacy through combined photothermal, photodynamic, and chemotherapy. The resulting nanocarriers showed excellent encapsulation capability for the hypoxia activatable prodrug tirapazamine (TPZ) and rapid drug release in the tumor sites. These vesicles could also produce hyperthermia and ROS upon laser irradiation at $660 \mathrm{~nm}$ for efficient anticancer therapy. The hypoxic microdomain owing to the oxygen consumption during the PDT process may further activate prodrug TPZ that would subsequently kill cancer cells as a chemotherapeutic agent. Therefore, this study offered an innovative method to create a multifunctional therapeutic nanoplatform that may have potential applications in improved anticancer therapy.

\section{WP[n]-based nanocarriers as optical materials}

\subsection{WP $[n]$-based nanocarriers for bioimaging}

$\mathrm{WP}[n]$-based nanocarriers with excellent cytocompatibility were also used as vehicles for bioimaging. For example, Zhao and co-workers fabricated a number of amphiphilic WP[5]s for delivering dye mixtures in dual-modality bioimaging. ${ }^{116}$ In 2016, Huang and co-workers developed NPs with NIR emission promoted by WP[5] 


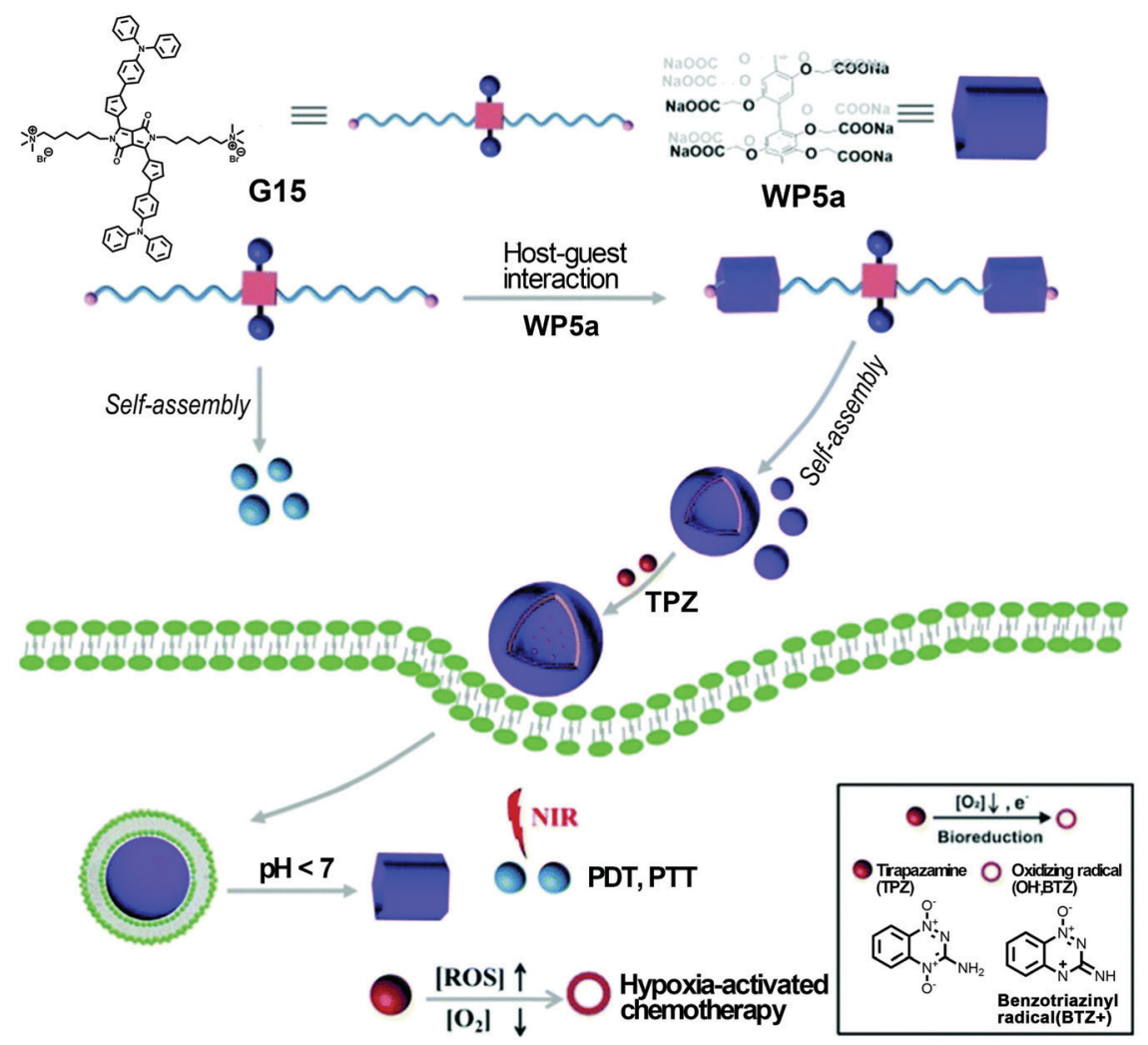

Fig. 19 Cartoon representation of fabricating multifunctional supramolecular vesicles based on WP5a and G15 for combined PDT/PTT/chemotherapy. Reproduced with permission from ref. 115. Copyright 2018, the Royal Society of Chemistry.

in aqueous media (Fig. 20). ${ }^{117}$ Cyanostilbene derivatives showed special fluorescent properties, which absorbed intensely in the visible region and emitted strongly in the range from red to NIR. In this work, they used the cyanostilbene derivative $\mathbf{G 1 6}$ as a building block in the fabrication of NIR emitting NPs. G16 alone is amphiphilic and can self-assemble into nanoribbons due to $\pi-$ $\pi$ stacking and hydrophobic interactions, exhibiting weak emission in aqueous media. The nanoribbons transformed into NPs in the presence of WP5b due to the formation of the WP5b $\supset$ G16 host-guest complex. The $K_{\mathrm{a}}$ value of WP5b and the model guest $\mathbf{G 1 7}$ was determined to be $(1.75 \pm 0.21) \times 10^{6} \mathrm{M}^{-1}$ by ITC. The obtained NPs exhibited strong NIR fluorescence due to the host-guest complexation-enhanced aggregation. MTT assays exhibited that the cell viability (HeLa cells and bEnd. 3 cells) of G16 was lower than that of WP5b $\supset$ G16, indicating that the formation of NPs reduced the cytotoxicity of G16. Furthermore, the NPs were used as an imaging agent for cells because of their NIR emission property. Both bEnd.3 cells and HeLa cells incubated with such nanocarriers showed enhanced red fluorescence inside the cytoplasm of cells. This study demonstrated that $\mathrm{WP}[n]$-based supra-amphiphiles had great potential in biological science, such as cellular imaging.

\subsection{WP $[n]$-based nanocarriers for artificial light harvesting} (ALH)

Inspired by the effectiveness of photosynthesis, the construction of an ALH system is becoming a promising research field.
In 2016, Diao and co-workers prepared light-harvesting antenna complexes by using $\mathrm{WP}[5]$ and $\beta$-carotene. ${ }^{118}$ In 2018, Yang and co-workers fabricated supramolecular polymers based on pillar[5]arene, which could further form ALH NPs. ${ }^{119}$ More recently, $\mathrm{Hu}$, Wang and co-workers constructed a new ALH system in an aqueous solution by employing WP6a (Fig. 21). ${ }^{120}$ In particular, this system was self-assembled from WP6a, a salicylaldehyde azine derivative guest (G18), and two fluorescent dyes, Eosin Y (ESY) or Nile Red (NiR). Guest G18 is strongly emissive when aggregated. In this study, G18 complexed with WP6a through host-guest recognition and further formed NPs by hydrophobic interaction. Notably, WP6a not only dramatically lowered the CAC of $\mathbf{G 1 8}$ but also remarkably improved the AIE of the guest. The CAC of the guest was decreased by at least 28-fold and the fluorescence intensity was enhanced 30 times, upon addition of WP6a. These NPs served as a donor for the ALH systems, while the hydrophobic dye NiR or ESY played the role of acceptors that were located in the hydrophobic interior of the nanocarriers. The fluorescence decay profile exhibited that energy transfer occurred between the supra-amphiphile and both ESY and NiR fluorophores. When excited at $365 \mathrm{~nm}$, the fluorescence at $513 \mathrm{~nm}$ of WP6a $\supset$ G18 decreased. Interestingly, the fluorescence at $619 \mathrm{~nm}$ (NiR) increased. It should be noted that the emission of free NiR was very weak when irradiated at $365 \mathrm{~nm}$. Further investigations exhibited that ESY acted as a good acceptor. Appealingly, the maximal antenna effects were calculated to be 

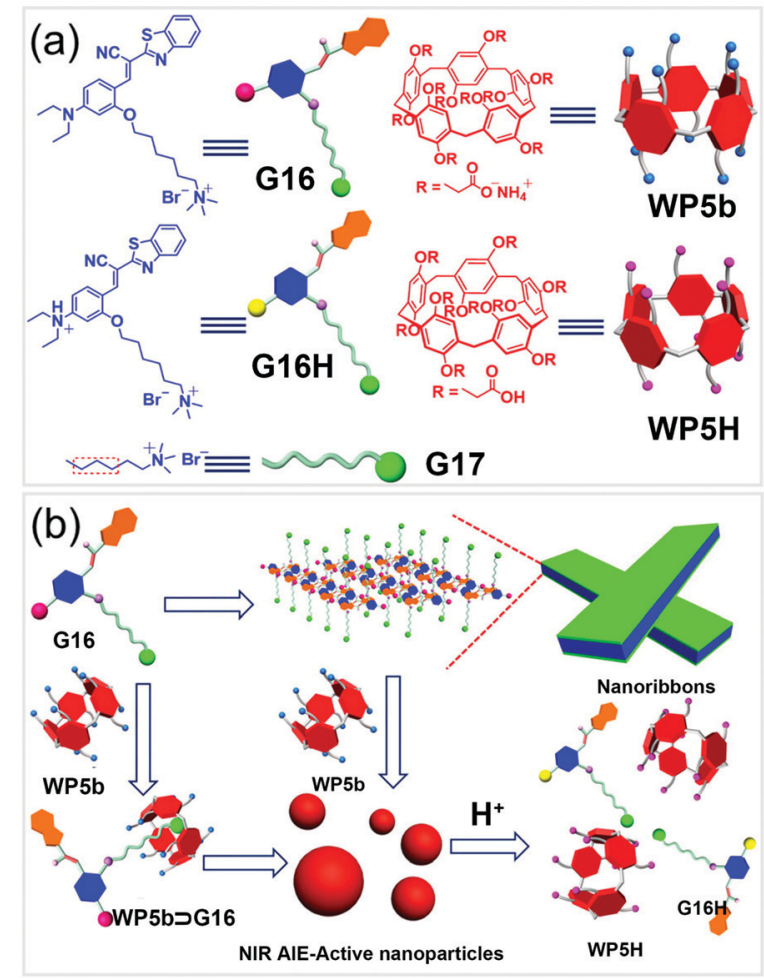

Fig. 20 (a) Chemical structures of the guests, WP5b, and WP5H, and (b) schematic illustration of self-assemblies of G16 and WP5b $\supset$ G16 and $\mathrm{pH}$ responsiveness of the NPs. Reproduced with permission from ref. 117. Copyright 2016, American Chemical Society.

28.0 for ESY and 25.4 for NiR, demonstrating that such NPs are excellent ALH antennas in aqueous media. This investigation provided an excellent artificial light harvesting system for mimicking photosynthesis.

\subsection{WP $[n]$-based nanocarriers for anti-counterfeiting inks}

The use of optical nanomaterials to develop anti-counterfeiting technology has attracted more and more attention. In 2018, $\mathrm{Hu}$, Jiang, Wang and co-workers reported full-color tunable fluorescent supramolecular nanocarriers that could be used as anti-counterfeiting inks (Fig. 22). ${ }^{121}$ Three types of copolymers were synthesized, namely polyfluorene (PF) containing dithienylbenzothiadiazole (DBT) blocks (G19), poly(fluorene-thienylene) (G20), and poly(fluorenephenylene) (G21). Notably, all of the polymer guests contain trimethylammonium units in the chains, which can bind with WP5a via host-guest interactions. Interestingly, WP5a $\supset$ G19, WP5a $\supset$ G20, and WP5a $\supset$ G21 produced single-band red, green, and blue fluorescence, respectively. Therefore, white-light was accessed by mixing WP5a with the three types of guest polymers in a special ratio. Morphology studies showed that all of these host-guest complexes formed NPs. Photo-responsive vesicles were constructed by the encapsulation of the dithienylethene derivative (DE) into the NPs. Subsequently, the fluorescence of the nanocarriers was quenched upon UV irradiation and recovered upon visible light irradiation, owing to FRET between the copolymer and the acceptor.

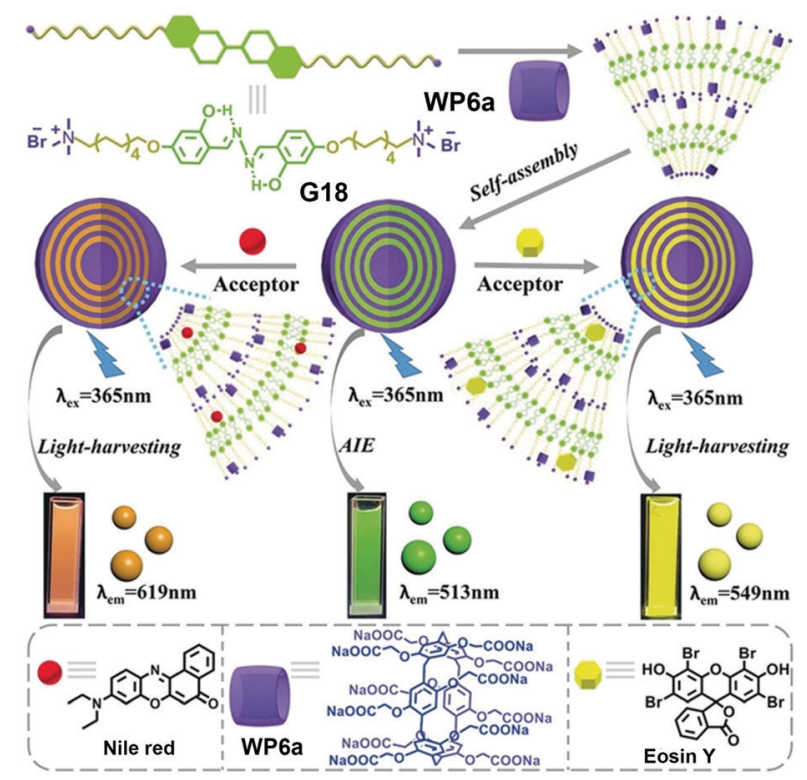

Fig. 21 Chemical structures of WP6a, G18, Nile red, Eosin Y, and illustration of the self-assembled aqueous ALH systems. Reproduced with permission from ref. 120. Copyright 2018, Wiley Publishers.

Moreover, a chemiluminescent material could be achieved by using a compound abbreviated as CPPO (Fig. 22) as a donor. As a result, the obtained materials can be employed as inks for potential anti-counterfeiting technology. Moreover, this work offered a novel strategy for protecting information by developing fluorescent dynamic nanomaterials based on $\mathrm{WP}[n] \mathrm{s}$.

\section{Conclusions and perspectives}

In summary, recent developments of stimuli-responsive supramolecular nanocarriers constructed from $\mathrm{WP}[n]$ have been reviewed. Preparation of these functional nanocarriers is generally straightforward. Two steps are usually required: (1) formation of supra-amphiphiles from a $\mathrm{WP}[n]$ and a hydrophobic guest via host-guest recognition; (2) self-assembly of supra-amphiphiles to form nanocarriers driven by hydrophobic interaction in aqueous media. Due to various functionalities of $\mathrm{WP}[n]$ and selected guest molecules, different supramolecular nanocarriers have been developed, including micelles, vesicles, and other types of NPs. More importantly, the intrinsic stimuli-responsive behaviors of $\mathrm{WP}[n]$ and/or guest species may endow the resultant nanocarriers with different responsiveness toward external stimuli, such as $\mathrm{pH}$, redox, light, GSH, and other chemical/physical stimuli, which has significantly expanded the applications of $\mathrm{WP}[n]$ based nanocarriers in many areas. In this review, we mainly described the potential applications of these nanocarriers in drug/gene/protein delivery, photodynamic/photothermal therapy, and optical materials.

Although many potential applications of supramolecular nanocarriers based on $\mathrm{WP}[n]$ have been demonstrated in recent years with significant progress, there are still many challenges that scientists in this research area are facing. For instance, a more practical platform that can be applied in the real world is 


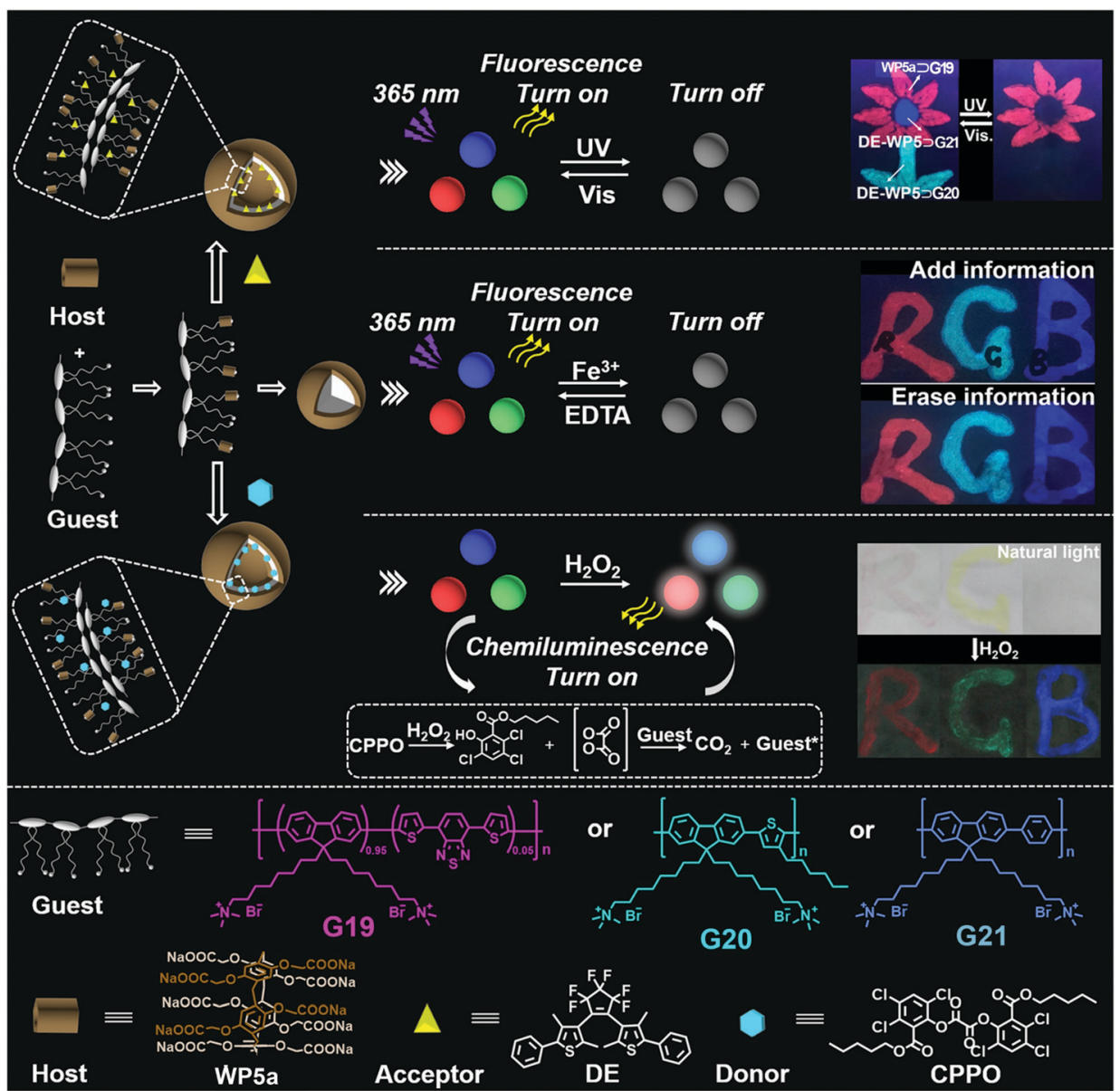

Fig. 22 Cartoon representation of the full-colour tunable fluorescent and chemiluminescent materials constructed from WP5a. Reproduced with permission from ref. 121. Copyright 2018, American Chemical Society.

still lacking. Here are a few points to tackle this issue: (1) in the future, supramolecular chemists may need more collaborations with computational chemists, material scientists, biologists, and clinicians in order to understand the real-world needs, and design and build suitable supramolecular nanocarriers geared for such needs; (2) for WP[n]-based nanocarriers used as potential DDSs, their systemic biocompatibility study in a mammal model via different administration routes (e.g. i.v., i.p., oral) is still missing. In particular, maximum tolerable dose (MTD) values need to be well defined in order to potentially translate these studies to large animal models or even clinical trials; (3) the study of combination therapy of WP $[n]$-based nanocarriers is still in its infancy. In addition to chemotherapy, PDT or PTT of $\mathrm{WP}[n]$-based nanocarriers can also be combined with magnetic hyperthermia therapy (MHT), immunotherapy, radiotherapy, or ultrasound therapy, for better treatment for cancer or diabetes; (4) the exploitation of $\mathrm{WP}[n]$-based nanocarriers as optical materials is still at the beginning stage. It is anticipated that more and more highly efficient optical materials, such as artificial light harvesting systems, will be developed in the future; (5) nanocarriers constructed from supra-amphiphiles based on other extended macrocyclic arenes, such as water-soluble derivatives of biphen $[n] \operatorname{arenes}^{122-124}$ and oxatub[4]arenes, ${ }^{125-127}$ might also be of interest to this research area. It is noteworthy that a water-soluble oxatub[4]arene has been prepared by Jiang and co-workers. ${ }^{128}$

As functional materials with potential applications in biomedical sciences, environmental sciences and energy are important research areas that scientists have pursued intensively. The practical applications of nanocarriers based on $\mathrm{WP}[n]$ in these aspects should continue be trendy research directions in the next decade. There is no doubt that further progress will be realized in these described fields due to the outstanding properties of $\mathrm{WP}[n] \mathrm{s}$ that have been discussed in this current review. Therefore, it can be expected that human society might greatly benefit from supramolecular nanocarriers, including those based on $\mathrm{WP}[n] \mathrm{s}$, in the next few decades.

\section{Conflicts of interest}

There are no conflicts to declare.

\section{Acknowledgements}

We gratefully acknowledge the financial support from the National Natural Science Foundation of China (No. 21702020 
and 21672102) and the Research Committee at University of Macau (MYRG2016-00165-ICMS-QRCM). Leyong Wang is grateful to the UM Macao Distinguished Visiting Scholar Scheme.

\section{Notes and references}

1 T. Xiao, W. Zhong, L. Zhou, L. Xu, X.-Q. Sun, R. B. P. Elmes, X.-Y. Hu and L. Wang, Chin. Chem. Lett., 2019, 30, 31-36.

2 M. A. Dobrovolskaia and S. E. McNeil, Nat. Nanotechnol., 2007, 2, 469-478.

3 T. M. Allen and P. R. Cullis, Science, 2004, 303, 1818-1822.

4 E. Rideau, R. Dimova, P. Schwille, F. R. Wurm and K. Landfester, Chem. Soc. Rev., 2018, 47, 8572-8610.

5 A. Sorrenti, O. Illa and R. M. Ortuño, Chem. Soc. Rev., 2013, 42, 8200-8219.

6 Y. Kang, X. Tang, Z. Cai and X. Zhang, Adv. Funct. Mater., 2016, 26, 8920-8931.

7 Y. Kang, K. Liu and X. Zhang, Langmuir, 2014, 30, 5989-6001.

8 X. Zhang and C. Wang, Chem. Soc. Rev., 2011, 40, 94-101.

9 Y. Chang, Y. Jiao, H. E. Symons, J.-F. Xu, C. F. J. Faul and X. Zhang, Chem. Soc. Rev., 2019, 48, 989-1003.

10 G. Yu, K. Jie and F. Huang, Chem. Rev., 2015, 115, 7240-7303.

11 H. Zhu, L. Shangguan, B. Shi, G. Yu and F. Huang, Mater. Chem. Front., 2018, 2, 2152-2174.

12 J.-R. Wu and Y.-W. Yang, Chem. Commun., 2019, 55, 1533-1543.

13 Y. Chen, F. Huang, Z.-T. Li and Y. Liu, Sci. China: Chem., 2018, 61, 979-992.

14 H. Xiong, D. Zhou, X. Zheng, Y. Qi, Y. Wang, X. Jing and Y. Huang, Chem. Commun., 2017, 53, 3422-3425.

15 Q. Pei, X. Hu, L. Wang, S. Liu, X. Jing and Z. Xie, ACS Appl. Mater. Interfaces, 2017, 9, 26740-26748.

16 Q. Yan, J. Yuan, Z. Cai, Y. Xin, Y. Kang and Y. Yin, J. Am. Chem. Soc., 2010, 132, 9268-9270.

17 C. Hu, N. Ma, F. Li, Y. Fang, Y. Liu, L. Zhao, S. Qiao, X. Li, X. Jiang, T. Li, F. Shen, Y. Huang, Q. Luo and J. Liu, ACS Appl. Mater. Interfaces, 2018, 10, 4603-4613.

18 C. Gao, Q. Huang, Q. Lan, Y. Feng, F. Tang, M. P. M. Hoi, J. Zhang, S. M. Y. Lee and R. Wang, Nat. Commun., 2018, 9, 2967.

19 Y. Yang, P. He, Y. Wang, H. Bai, S. Wang, J.-F. Xu and X. Zhang, Angew. Chem., Int. Ed., 2017, 56, 16239-16242.

20 D. Jiao, J. Geng, X. J. Loh, D. Das, T.-C. Lee and O. A. Scherman, Angew. Chem., Int. Ed., 2012, 51, 9633-9637.

21 H. Yin and R. Wang, Isr. J. Chem., 2018, 58, 188-198.

22 Z. Xu, S. Jia, W. Wang, Z. Yuan, B. Jan Ravoo and D.-S. Guo, Nat. Chem., 2019, 11, 86-93.

23 X.-M. Chen, Y. Chen, Q. Yu, B.-H. Gu and Y. Liu, Angew. Chem., Int. Ed., 2018, 57, 12519-12523.

24 Y.-X. Wang, Y.-M. Zhang and Y. Liu, J. Am. Chem. Soc., 2015, 137, 4543-4549.

25 D.-S. Guo, K. Wang, Y.-X. Wang and Y. Liu, J. Am. Chem. Soc., 2012, 134, 10244-10250.
26 X. Ji, S. Dong, P. Wei, D. Xia and F. Huang, Adv. Mater., 2013, 25, 5725-5729.

27 X. Ji, J. Li, J. Chen, X. Chi, K. Zhu, X. Yan, M. Zhang and F. Huang, Macromolecules, 2012, 45, 6457-6463.

28 T. Kakuta, T.-a. Yamagishi and T. Ogoshi, Acc. Chem. Res., 2018, 51, 1656-1666.

29 T. Ogoshi, T.-a. Yamagishi and Y. Nakamoto, Chem. Rev., 2016, 116, 7937-8002.

30 N. L. Strutt, H. Zhang, S. T. Schneebeli and J. F. Stoddart, Acc. Chem. Res., 2014, 47, 2631-2642.

31 M. Xue, Y. Yang, X. Chi, Z. Zhang and F. Huang, Acc. Chem. Res., 2012, 45, 1294-1308.

32 D. Cao, Y. Kou, J. Liang, Z. Chen, L. Wang and H. Meier, Angew. Chem., Int. Ed., 2009, 48, 9721-9723.

33 T. Ogoshi, S. Kanai, S. Fujinami, T.-A. Yamagishi and Y. Nakamoto, J. Am. Chem. Soc., 2008, 130, 5022-5023.

34 K. Yang, Y. Pei, J. Wen and Z. Pei, Chem. Commun., 2016, 52, 9316-9326.

35 W. L. Imlach, R. F. Bhola, S. A. Mohammadi and M. J. Christie, Sci. Rep., 2016, 6, 37104.

36 P. Li, Y. Chen and Y. Liu, Chin. Chem. Lett., 2019, 30, 1190-1197.

37 Y. Wang, G. Ping and C. Li, Chem. Commun., 2016, 52, 9858-9872.

38 T. Xiao, L. Xu, W. Zhong, L. Zhou, X.-Q. Sun, X.-Y. Hu and L. Wang, Isr. J. Chem., 2018, 58, 1183-1193.

39 T. Xiao, L. Xu, L. Zhou, X.-Q. Sun, C. Lin and L. Wang, J. Mater. Chem. B, 2019, 7, 1526-1540.

40 T. Xiao and L. Wang, Tetrahedron Lett., 2018, 59, 1172-1182.

41 T. Xiao, L. Zhou, L. Xu, W. Zhong, W. Zhao, X.-Q. Sun and R. B. P. Elmes, Chin. Chem. Lett., 2019, 30, 271-276.

42 S. Dong, J. Yuan and F. Huang, Chem. Sci., 2014, 5, 247-252.

43 Q. Lin, X.-W. Guan, S.-S. Song, H. Fan, H. Yao, Y.-M. Zhang and T.-B. Wei, Polym. Chem., 2019, 10, 253-259.

44 Y.-M. Zhang, Y.-F. Li, H. Fang, J.-X. He, B.-R. Yong, H. Yao, T.-B. Wei and Q. Lin, Soft Matter, 2018, 14, 8529-8536.

45 Z.-Y. Li, Y. Zhang, C.-W. Zhang, L.-J. Chen, C. Wang, H. Tan, Y. Yu, X. Li and H.-B. Yang, J. Am. Chem. Soc., 2014, 136, 8577-8589.

46 Q. Wang, P. Zhang, Y. Li, L. Tian, M. Cheng, F. Lu, X. Lu, Q. Fan and W. Huang, RSC Adv., 2017, 7, 29364-29367.

47 Q. Wang, M. Cheng, L. Tian, Q. Fan and J. Jiang, Polym. Chem., 2017, 8, 6058-6063.

48 T. Ogoshi, H. Kayama, D. Yamafuji, T. Aoki and T.-a. Yamagishi, Chem. Sci., 2012, 3, 3221-3226.

49 T. Xiao, L. Zhou, X.-Q. Sun, F. Huang, C. Lin and L. Wang, Chin. Chem. Lett., 2019, DOI: 10.1016/j.cclet.2019.05.011.

50 T. Xiao, L. Xu, J. Wang, Z.-Y. Li, X.-Q. Sun and L. Wang, Org. Chem. Front., 2019, 6, 936-941.

51 T. Xiao, W. Zhong, L. Qi, J. Gu, X. Feng, Y. Yin, Z.-Y. Li, X.-Q. Sun, M. Cheng and L. Wang, Polym. Chem., 2019, 10, 3342-3350.

52 D. Dai, Z. Li, J. Yang, C. Wang, J.-R. Wu, Y. Wang, D. Zhang and Y. W. Yang, J. Am. Chem. Soc., 2019, 141, 4756-4763.

53 W. Si, P. Xin, Z.-T. Li and J.-L. Hou, Acc. Chem. Res., 2015, 48, 1612-1619. 
54 K. Jie, Y. Zhou, E. Li and F. Huang, Acc. Chem. Res., 2018, 51, 2064-2072.

55 K. Jie, Y. Zhou, E. Li, R. Zhao, M. Liu and F. Huang, J. Am. Chem. Soc., 2018, 140, 3190-3193.

56 K. Jie, Y. Zhou, E. Li, R. Zhao and F. Huang, Angew. Chem., Int. Ed., 2018, 57, 12845-12849.

57 K. Jie, M. Liu, Y. Zhou, M. A. Little, A. Pulido, S. Y. Chong, A. Stephenson, A. R. Hughes, F. Sakakibara, T. Ogoshi, F. Blanc, G. M. Day, F. Huang and A. I. Cooper, J. Am. Chem. Soc., 2018, 140, 6921-6930.

58 J. Chen, Y. Wang, C. Wang, R. Long, T. Chen and Y. Yao, Chem. Commun., 2019, 55, 6817-6826.

59 Q. Yao, B. Lü, C. Ji, Y. Cai and M. Yin, ACS Appl. Mater. Interfaces, 2017, 9, 36320-36326.

60 H. Zhang, Z. Liu and Y. Zhao, Chem. Soc. Rev., 2018, 47, 5491-5528.

61 W. Feng, M. Jin, K. Yang, Y. Pei and Z. Pei, Chem. Commun., 2018, 54, 13626-13640.

62 C. Sathiyajith, R. R. Shaikh, Q. Han, Y. Zhang, K. Meguellati and Y.-W. Yang, Chem. Commun., 2017, 53, 677-696.

63 T. Xiao, W. Zhong, L. Xu, X.-Q. Sun, X.-Y. Hu and L. Wang, Org. Biomol. Chem., 2019, 17, 1336-1350.

64 N. Song, X.-Y. Lou, L. Ma, H. Gao and Y.-W. Yang, Theranostics, 2019, 9, 3075-3093.

65 G. Yu, M. Xue, Z. Zhang, J. Li, C. Han and F. Huang, J. Am. Chem. Soc., 2012, 134, 13248-13251.

66 G. Yu, X. Zhou, Z. Zhang, C. Han, Z. Mao, C. Gao and F. Huang, J. Am. Chem. Soc., 2012, 134, 19489-19497.

67 Z. Li, J. Yang, G. Yu, J. He, Z. Abliz and F. Huang, Org. Lett., 2014, 16, 2066-2069.

68 D. Xia, G. Yu, J. Li and F. Huang, Chem. Commun., 2014, 50, 3606-3608.

69 J. Yang, G. Yu, D. Xia and F. Huang, Chem. Commun., 2014, 50, 3993-3995.

70 P. Li, Q. Yao, B. Lü, G. Ma and M. Yin, Macromol. Rapid Commun., 2018, 39, 1800133.

71 S. Guo, X. Liu, C. Yao, C. Lu, Q. Chen, X.-Y. Hu and L. Wang, Chem. Commun., 2016, 52, 10751-10754.

72 X. Chi, G. Yu, X. Ji, Y. Li, G. Tang and F. Huang, ACS Macro Lett., 2015, 4, 996-999.

73 Y.-H. Cui, R. Deng, Z. Li, X.-S. Du, Q. Jia, X.-H. Wang, C.-Y. Wang, K. Meguellati and Y.-W. Yang, Mater. Chem. Front., 2019, 3, 1427-1432.

74 X. Chi, X. Ji, D. Xia and F. Huang, J. Am. Chem. Soc., 2015, 137, 1440-1443.

75 X. Chi, G. Yu, L. Shao, J. Chen and F. Huang, J. Am. Chem. Soc., 2016, 138, 3168-3174.

76 J. Yang, L. Shao and G. Yu, Chem. Commun., 2016, 52, 3211-3214.

77 S. Wang, C. Yao, M. Ni, Z. Xu, M. Cheng, X.-Y. Hu, Y. Shen, C. Lin, L. Wang and D. Jia, Polym. Chem., 2017, 8, 682-688.

78 L. Jiang, X. Huang, D. Chen, H. Yan, X. Li and X. Du, Angew. Chem., Int. Ed., 2017, 56, 2655-2659.

79 X. Wu, L. Gao, X.-Y. Hu and L. Wang, Chem. Rec., 2016, 16, 1216-1227.
80 G. Sun, Z. He, M. Hao, M. Zuo, Z. Xu, X.-Y. Hu, J.-J. Zhu and L. Wang, J. Mater. Chem. B, 2019, 7, 3944-3949.

81 W. Xia, X.-Y. Hu, Y. Chen, C. Lin and L. Wang, Chem. Commun., 2013, 49, 5085-5087.

82 Q. Duan, Y. Cao, Y. Li, X.-Y. Hu, T. Xiao, C. Lin, Y. Pan and L. Wang, J. Am. Chem. Soc., 2013, 135, 10542-10549.

83 Y. Cao, X.-Y. Hu, Y. Li, X. Zou, S. Xiong, C. Lin, Y.-Z. Shen and L. Wang, J. Am. Chem. Soc., 2014, 136, 10762-10769.

84 X.-Y. Hu, K. Jia, Y. Cao, Y. Li, S. Qin, F. Zhou, C. Lin, D. Zhang and L. Wang, Chem. - Eur. J., 2015, 21, 1208-1220.

85 X. Wu, Y. Li, C. Lin, X.-Y. Hu and L. Wang, Chem. Commun., 2015, 51, 6832-6835.

86 X.-Y. Hu, X. Liu, W. Zhang, S. Qin, C. Yao, Y. Li, D. Cao, L. Peng and L. Wang, Chem. Mater., 2016, 28, 3778-3788.

87 Y. Yao, X. Chi, Y. Zhou and F. Huang, Chem. Sci., 2014, 5, 2778 .

88 D. Xia, L. Shangguan, M. Xue and B. Shi, New J. Chem., 2016, 40, 9890-9894.

89 X. Liu, K. Jia, Y. Wang, W. Shao, C. Yao, L. Peng, D. Zhang, X.-Y. Hu and L. Wang, ACS Appl. Mater. Interfaces, 2017, 9, 4843-4850.

90 S. Guo, T. Liang, Y. Song, M. Cheng, X.-Y. Hu, J.-J. Zhu and L. Wang, Polym. Chem., 2017, 8, 5718-5725.

91 G. Yu, R. Zhao, D. Wu, F. Zhang, L. Shao, J. Zhou, J. Yang, G. Tang, X. Chen and F. Huang, Polym. Chem., 2016, 7, 6178-6188.

92 G. Yu, W. Yu, L. Shao, Z. Zhang, X. Chi, Z. Mao, C. Gao and F. Huang, Adv. Funct. Mater., 2016, 26, 8999-9008.

93 Y. Cao, Y. Li, X.-Y. Hu, X. Zou, S. Xiong, C. Lin and L. Wang, Chem. Mater., 2015, 27, 1110-1119.

94 Y. Wang, J. Du, Y. Wang, Q. Jin and J. Ji, Chem. Commun., 2015, 51, 2999-3002.

95 W. Shao, X. Liu, G. Sun, X.-Y. Hu, J.-J. Zhu and L. Wang, Chem. Commun., 2018, 54, 9462-9465.

96 X.-Y. Hu, L. Gao, S. Mosel, M. Ehlers, E. Zellermann, H. Jiang, S. K. Knauer, L. Wang and C. Schmuck, Small, 2018, 14, 1803952.

97 K. Yang, Y. Chang, J. Wen, Y. Lu, Y. Pei, S. Cao, F. Wang and Z. Pei, Chem. Mater., 2016, 28, 1990-1993.

98 Y. Chang, C. Hou, J. Ren, X. Xin, Y. Pei, Y. Lu, S. Cao and Z. Pei, Chem. Commun., 2016, 52, 9578-9581.

99 K. Yang, K. Yang, S. Chao, J. Wen, Y. Pei and Z. Pei, Chem. Commun., 2018, 54, 9817-9820.

100 Y. Chang, K. Yang, P. Wei, S. Huang, Y. Pei, W. Zhao and Z. Pei, Angew. Chem., Int. Ed., 2014, 53, 13126-13130.

101 L. Gao, T. Wang, K. Jia, X. Wu, C. Yao, W. Shao, D. Zhang, X.-Y. Hu and L. Wang, Chem. - Eur. J., 2017, 23, 6605-6614.

102 M. Zuo, W. Qian, Z. Xu, W. Shao, X. Y. Hu, D. Zhang, J. Jiang, X. Sun and L. Wang, Small, 2018, 14, 1801942.

103 K. Yang, J. Wen, S. Chao, J. Liu, K. Yang, Y. Pei and Z. Pei, Chem. Commun., 2018, 54, 5911-5914.

104 J. F. Lovell, T. W. B. Liu, J. Chen and G. Zheng, Chem. Rev., 2010, 110, 2839-2857.

105 H. Kim, S. Beack, S. Han, M. Shin, T. Lee, Y. Park, K. S. Kim, A. K. Yetisen, S. H. Yun, W. Kwon and S. K. Hahn, Adv. Mater., 2018, 30, 1701460. 
106 Y. Jiang and K. Pu, Acc. Chem. Res., 2018, 51, 1840-1849. 107 M.-X. Wu, H.-J. Yan, J. Gao, Y. Cheng, J. Yang, J.-R. Wu, B.-J. Gong, H.-Y. Zhang and Y.-W. Yang, ACS Appl. Mater. Interfaces, 2018, 10, 34655-34663.

108 L. Rui, Y. Xue, Y. Wang, Y. Gao and W. Zhang, Chem. Commun., 2017, 53, 3126-3129.

109 Y. Chen, L. Rui, L. Liu and W. Zhang, Polym. Chem., 2016, 7, 3268-3276.

110 H. Zhu, H. Wang, B. Shi, L. Shangguan, W. Tong, G. Yu, Z. Mao and F. Huang, Nat. Commun., 2019, 10, 2412.

111 L.-B. Meng, W. Zhang, D. Li, Y. Li, X.-Y. Hu, L. Wang and G. Li, Chem. Commun., 2015, 51, 14381-14384.

112 S. Goel, F. Chen and W. Cai, Small, 2014, 10, 631-645.

113 Q.-L. Li, Y. Sun, L. Ren, X. Wang, C. Wang, L. Li, Y.-W. Yang, X. Yu and J. Yu, ACS Appl. Mater. Interfaces, 2018, 10, 29314-29324.

114 Q. Wang, P. Zhang, J. Xu, B. Xia, L. Tian, J. Chen, J. Li, F. Lu, Q. Shen, X. Lu, W. Huang and Q. Fan, ACS Appl. Bio Mater., 2018, 1, 70-78.

115 Q. Wang, L. Tian, J. Xu, B. Xia, J. Li, F. Lu, X. Lu, W. Wang, W. Huang and Q. Fan, Chem. Commun., 2018, 54, 10328-10331.

116 H. Zhang, X. Ma, K. T. Nguyen and Y. Zhao, ACS Nano, 2013, 7, 7853-7863.
117 B. Shi, K. Jie, Y. Zhou, J. Zhou, D. Xia and F. Huang, J. Am. Chem. Soc., 2016, 138, 80-83.

118 Y. Sun, F. Guo, T. Zuo, J. Hua and G. Diao, Nat. Commun., 2016, 7, 12042.

119 C.-L. Sun, H.-Q. Peng, L.-Y. Niu, Y.-Z. Chen, L.-Z. Wu, C.-H. Tung and Q.-Z. Yang, Chem. Commun., 2018, 54, 1117-1120.

120 S. Guo, Y. Song, Y. He, X.-Y. Hu and L. Wang, Angew. Chem., Int. Ed., 2018, 57, 3163-3167.

121 M. Zuo, W. Qian, T. Li, X.-Y. Hu, J. Jiang and L. Wang, ACS Appl. Mater. Interfaces, 2018, 10, 39214-39221.

122 B. Li, B. Wang, X. Huang, L. Dai, L. Cui, J. Li, X. Jia and C. Li, Angew. Chem., Int. Ed., 2019, 58, 3885-3889.

123 J. Ma, H. Deng, S. Ma, J. Li, X. Jia and C. Li, Chem. Commun., 2015, 51, 6621-6624.

124 H. Chen, J. Fan, X. Hu, J. Ma, S. Wang, J. Li, Y. Yu, X. Jia and C. Li, Chem. Sci., 2015, 6, 197-202.

125 H. Ke, L. P. Yang, M. Xie, Z. Chen, H. Yao and W. Jiang, Nat. Chem., 2019, 11, 470-477.

126 F. Jia, D.-H. Li, T.-L. Yang, L.-P. Yang, L. Dang and W. Jiang, Chem. Commun., 2017, 53, 336-339.

127 F. Jia, Z. He, L.-P. Yang, Z.-S. Pan, M. Yi, R.-W. Jiang and W. Jiang, Chem. Sci., 2015, 6, 6731-6738.

128 L.-P. Yang, H. Liu, S.-B. Lu, F. Jia and W. Jiang, Org. Lett., 2017, 19, 1212-1215. 